\title{
PSEUDO P-POINTS AND SPLITTING NUMBER
}

\author{
ALAN DOW AND SAHARON SHELAH
}

AbStract. We construct a model in which the splitting number is large and every ultrafilter has a small subset with no pseudo-intersection.

Throughout the paper, $\operatorname{Hyp}(\kappa, \lambda)$ will denote the assumptions detailed in this paragraph. Each of $\kappa$ and $\lambda$ is a regular cardinal and $\aleph_{1}<\kappa<\lambda, \lambda^{<\lambda}=\lambda$. The set $E$ is a stationary subset of $S_{\kappa}^{\lambda}$ where $S_{\kappa}^{\lambda} \subset \lambda$ is the set of ordinals of cofinality $\kappa$. There is a $\square$-sequence $\left\{C_{\alpha}: \alpha \in \lambda\right\}$ such that for limit ordinals $\alpha<\beta \in \lambda$

(1) $C_{\alpha}$ is a closed unbounded subset of $\alpha$,

(2) if $\alpha \in \operatorname{acc}\left(C_{\beta}\right)$, then $C_{\alpha}=C_{\beta} \cap \alpha$,

(3) $C_{\alpha} \cap E$ is empty.

Naturally $E$ is a non-reflecting stationary set. We also assume there is a $\diamond(E)$ sequence $\left\{X_{\alpha}: \alpha \in E\right\}$, where $X_{\alpha} \subset \alpha$ and for all $X \subset \lambda$, there is a stationary set $E_{X} \subset E$ such that $X_{\alpha}=X \cap \alpha$ for all $\alpha \in E_{X}$.

A set $b \subset \mathbb{N}$ is a pseudo-intersection of a family $\mathcal{A}$ of subsets of $\mathbb{N}$, if $b$ is infinite and $b \backslash a$ is finite for all $a \in \mathcal{A}$. The pseudo-intersection number of a free ultrafilter $\mathcal{U}$ on $\mathbb{N}$, denoted $\pi p(\mathcal{U})$ is the smallest cardinal $\mu$ such that there is a subset $\mathcal{A} \subset \mathcal{U}$ of cardinality $\mu$ with no pseudo-intersection. The splitting number, $\mathfrak{s}$, is very well known. It can be defined as the minimum cardinal such that for every family $\mathcal{A} \subset[\mathbb{N}]^{\aleph_{0}}$ of smaller cardinality, there is a maximal free filter on the Boolean algebra generated by $\mathcal{A} \cup[\mathbb{N}]<\aleph_{0}$ that has a pseudo-intersection.

It was shown in [6] that it is consistent to have $\pi p(\mathcal{U})^{+} \leq \mathfrak{s}$ for all free ultrafilters $\mathcal{U}$ on $\mathbb{N}$. We construct a model by ccc forcing in which $\mathfrak{s}=\lambda=\mathfrak{c}$ and $\pi p(\mathcal{U}) \leq \kappa$ for all ultrafilters $\mathcal{U}$ on $\mathbb{N}$. In fact we construct two models, one in which $\mathfrak{b}=\lambda$ and the second in which $\mathfrak{b}=\kappa$.

\section{PRELIMINARIES}

For a poset $\left(P,<_{P}\right)$, a set $D \subset P$ is dense if for each $p \in P$, there is a $d \in D$ with $p<d$. Similarly, a set $G \subset P$ is a filter (using the Jerusalem convention) if it is closed downwards and finitely directed upwards. Therefore in the forcing language if $p<q$ are in $P, q$ is a stronger condition and a subset $A$ of $P$ is an antichain if no pair of elements of $A$ have a common upper bound. For convenience, we assume each forcing poset has a minimum element $1_{P}$.

A $P$-name $\dot{a}$ of a subset of $\omega$ (respectively $\mathbb{N}$ ) is canonical if for each $n \in \omega$ (respectively $n \in \mathbb{N}$ ), there is a (possibly empty) antichain $A_{n}$ of $P$ such that $\dot{a}=\bigcup\left\{\{n\} \times A_{n}: n \in \omega\right\}$. There should be no risk of confusion if we abuse notation and let each $n \in \omega$ also denote a $P$-name for itself.

For an infinite set $I$, the poset $\operatorname{Fn}(I, 2)$ is the standard Cohen poset consisting of finite partial functions from $I$ into 2 ordered by extension. If $G$ is a generic filter

Research partially supported by NSF grant no: 136974. Paper 1134 on Shelah's list. 
for $\operatorname{Fn}(I \times \mathbb{N}, 2)$, we obtain the standard sequence $\left\{\dot{x}_{i}: i \in I\right\}$ of canonical names for Cohen reals where $\dot{x}_{i}=\{(n,\langle(i, n), 1\rangle): n \in \mathbb{N}\}$. We will refer to this sequence as the canonical generic sequence we get from $\operatorname{Fn}(I \times \mathbb{N}, 2)$. This family is forced to have the finite intersection property, moreover, it is forced to be an independent family. However, rather than design a new poset we can use such sequences to define a uncountable families of pairwise almost disjoint subsets that are each Cohen over the ground model. Fix a sequence $\left\{e_{\alpha}: \alpha \in \omega_{1}\right\}$ (in the ground model) so that for each $\omega \leq \alpha \in \omega_{1}, e_{\alpha}$ is a bijection from $\omega$ onto $\alpha$.

Definition 1.1. For any sequence $\vec{x}=\left\{x_{\alpha}: \alpha \in \omega_{1}\right\}$ of subsets of $\mathbb{N}$, define, for $\alpha \in \omega_{1}, c(\vec{x}, \alpha)$ and $a(\vec{x}, \alpha)$ where

(1) for $\alpha<\omega, c(\vec{x}, \alpha)=x_{\alpha} \backslash \bigcup_{k<\alpha} x_{k}$,

(2) for $\omega \leq \alpha, c(\vec{x}, \alpha)=\left\{\min \left(x_{\alpha} \backslash \bigcup\left\{x_{e_{\alpha}(k)}: k<n\right\}\right): n \in \omega\right\}$,

(3) $a(\vec{x}, \alpha)=\mathbb{N} \backslash c(\vec{x}, \alpha)$.

Definition 1.2. For any set $I$, we have the canonical generic sequence $\left\{\dot{x}_{i, \alpha}\right.$ : $\left.i \in I, \alpha \in \omega_{1}\right\}$ for the poset $\operatorname{Fn}\left(I \times \omega_{1} \times N, 2\right)$. For each $i \in I$, we will let $\vec{x}_{i}$ denote the subsequence $\left\{\dot{x}_{i, \alpha}: \alpha \in \omega_{1}\right\}$. We then similarly have the sequences $\left\{\dot{c}\left(\vec{x}_{i}, \alpha\right): \alpha \in \omega_{1}\right\}$ and $\left\{\dot{a}\left(\vec{x}_{i}, \alpha\right): \alpha \in \omega_{1}\right\}$ defined as in Definition 1.1.

Let us recall that a poset $\left(P,<_{P}\right)$ is a complete suborder of a poset $\left(Q,<_{Q}\right)$ providing $P \subset Q,<_{P} \subset<_{Q}$, and each maximal antichain of $\left(P,<_{P}\right)$ is also a maximal antichain of $\left(Q,<_{Q}\right)$. Note that it follows that incomparable members of $\left(P,<_{P}\right)$ are still incomparable in $\left(Q,<_{Q}\right)$, i.e. $p_{1} \perp_{P} p_{2}$ implies $p_{1} \perp_{Q} p_{2}$. We will say that a chain $\left\{P_{i}: i<\kappa\right\}$ of posets is a $<-$-chain of posets if $P_{i}<\cdot P_{j}$ for all $i<j<\kappa$. We will say such a chain is a continuous <--chain if $P_{j}=\bigcup_{i<j} P_{i}$ whenever $j$ has uncountable cofinality. We will use the term strongly continuous for a chain $\left\{P_{\alpha}: \alpha<\gamma\right\}$ of posets if $P_{\beta}=\bigcup_{\alpha<\beta} P_{\alpha}$ for all limits $\beta<\gamma$.

Proposition 1.3. If $P<\cdot Q$ and $q \in Q$, then there is a $p \in P$ (a projection) with the property that for all $r \in P$ with $p<_{P} r$ ( $r$ is stronger than $p$ ), there is a $q_{r} \in Q$ that is stronger than each of $q$ and $r$.

When we say that $V$ or $V^{\prime}$ is a model, we will mean a transitive set that is a model of a sufficiently large fragment of ZFC.

Definition 1.4. Let $V$ and $V^{\prime}$ be models with $V \subset V^{\prime}$.

(1) If $P \in V$ and $Q \in V^{\prime}$ are posets, we write $P<_{V} Q$ if $P \subset Q,<_{P} \subset<_{Q}$, and each maximal antichain $A \subset P$ in $V$ is also a maximal antichain of $Q$. Of course $P<_{V^{\prime}} Q$ is the same as $V^{\prime} \models P<\cdot Q$.

(2) A family $\mathcal{A} \subset[\mathbb{N}]^{\aleph_{0}}$ is thin over $V$ if for each $\ell \in \omega$ and each infinite sequence $\left\{H_{n}: n \in \omega\right\} \subset[\mathbb{N}] \leq \ell \cap V$ of pairwise disjoint sets, there is, for each $a$ in the ideal generated by $\mathcal{A}$, an $n$ such that $H_{n} \cap a$ is empty.

(3) A family $\mathcal{A} \subset[\mathbb{N}]^{\aleph_{0}}$ is very thin over $V$ if for each a in the ideal generated by $\mathcal{A}$ and each $g \in \mathbb{N}^{\mathbb{N}} \cap V$, there is an $n \in \mathbb{N}$ such that $a \cap[n, g(n)]$ is empty.

We also will need the next result taken from [5, Lemma 13].

Lemma 1.5. Let $\mathbb{P}, \mathbb{Q}$ be partial orders such that $\mathbb{P}<\cdot \mathbb{Q}$. Recall that the name $\operatorname{id}_{P}=\{(p, p): p \in P\}$ is the $P$-name for the generic filter on $P$. Let $\dot{\mathbb{A}}$ be a $\mathbb{P}$-name for a forcing notion and let $\mathbb{B}$ be a $\mathbb{Q}$-name for a forcing notion such that $\vdash_{\mathbb{Q}} \dot{\mathbb{A}}<_{V\left[\mathrm{id}_{P}\right]} \dot{\mathbb{B}}$, then $\mathbb{P} * \dot{\mathbb{A}}<\cdot \mathbb{Q} * \dot{\mathbb{B}}$ 
It is immediate that the conclusion of Lemma 1.5 holds if $\mathbb{P}$ forces that either $\mathbb{A}=\mathbb{B}$ or if $\mathbb{A}=\operatorname{Fn}(I, 2) \subset \operatorname{Fn}(J, 2)=\mathbb{B}$.

Proposition 1.6. If $V \subset V^{\prime}$ are models and $\mathcal{A}$ is thin (very thin) over $V$, then for each $\alpha \in \omega_{1}, \mathcal{A} \cup\{a(\vec{x}, \alpha)\}$ is thin (respectively very thin) over $V$ where $\vec{x}=\left\{\dot{x}_{\alpha}\right.$ : $\left.\alpha \in \omega_{1}\right\}$ is the canonical generic sequence we get from forcing with $\operatorname{Fn}\left(\omega_{1} \times \mathbb{N}, 2\right)$ over $V$.

Proof. Fix any $a$ in the ideal generated by $\mathcal{A}$ and let $\left\{H_{n}: n \in \omega\right\} \subset[\mathbb{N}]<\aleph_{0}$ be any pairwise disjoint family in $V$. Let $p \in \operatorname{Fn}(\omega \times \mathbb{N}, 2)$ be any condition and assume that $\left\{n \in \omega: a \cap H_{n}\right\}$ is infinite. It suffices to prove that there is a $q$ extending $p$ and an $n$ such that $a \cap H_{n}=\emptyset$ and $q \Vdash H_{n} \cap \dot{a}(\vec{x}, \alpha)=\emptyset$. We will skip the case when $\alpha<\omega$ since it is easier. Choose a finite set $F \subset \omega_{1}$ and an integer $m \in \mathbb{N}$ such that $\operatorname{dom}(p) \subset F \times\{1, \ldots, m\}$. By extending $F$ but not $m$, we can assume that $\left\{e_{\alpha}(k): k<m\right\} \subset F$ and $\operatorname{dom}(p)=F \times\{1, \ldots, m\}$. Choose $n$ so that $m<\min \left(H_{n}\right)$ and $H_{n} \cap a=\emptyset$. We define an extension $q$ of $p$ that forces that $H_{n} \subset c(\vec{x}, \alpha)$. Let $\ell=\max \left(H_{n}\right)+m$ and $F^{\prime}=F \cup\left\{e_{\alpha}(k): k \leq \ell\right\}$. Define $q \supset p$ so that for all $(\beta, j) \in F^{\prime} \times\{1, \ldots, \ell\} \backslash \operatorname{dom}(p), q(\beta, j)=1$ if and only if $\beta=\alpha$. It is immediate that $q \Vdash[m+1, \ell] \cap \dot{x}_{e_{\alpha}(k)}=\emptyset$ for all $k<\ell$. Similarly, $q \Vdash[m+1, \ell] \subset \dot{x}_{\alpha}$. It follows that there is a $j_{0} \leq m$ such that $m+1$ is forced by $q$ to be the minimum element of $\dot{x}_{\alpha} \backslash \bigcup\left\{\dot{x}_{e_{\alpha}(k)}: k<j_{0}\right\}$. Then, by induction on $1<j<\max \left(H_{n}\right), m+j$ is forced by $q$ to be the minimum element of $\dot{x}_{\alpha} \backslash \bigcup\left\{\dot{x}_{e_{\alpha}(k)}: k<j_{0}+j\right\}$.

Definition 1.7. For a poset $P$ and infinite set $X$, let $\wp(X, P)$ denote the set of canonical names of infinite subsets of $X$ (meaning $1_{P}$ forces that each $\dot{a} \in \wp(X, P)$ is infinite). When $\mathcal{E}$ is a subset of $\wp(X, P)$ we will use it in forcing statements to mean the $P$-name $\left\{\left(\dot{a}, 1_{P}\right): \dot{a} \in \mathcal{E}\right\}$.

Proposition 1.8. If $\left\{P_{i}: i<\kappa\right\}$ is a continuous $<\cdot-$ chain of ccc posets and if $\dot{Q}_{i}$ is the $P_{i}$-name for the poset $\operatorname{Fn}(i+1 \times \theta \times \mathbb{N}, 2)$ for any ordinal $\theta$, then $\left\langle P_{i} * \dot{Q}_{i}: i<\kappa\right\rangle$ is a continuous $<-$-chain. If, in addition, $\left\{\mathcal{A}_{i}: i<\kappa\right\}$ is a sequence such that, for each $i<\kappa$,

(1) $\mathcal{A}_{i} \subset \wp\left(\mathbb{N}, P_{i+1}\right)$,

(2) $\mathcal{A}_{i}$ is forced (by $P_{i+1}$ ) to be thin (respectively very thin) over the forcing extension by $P_{i}$,

then, for each $i<\kappa, \mathcal{A}_{i}$ is forced to be thin (respectively very thin) over the forcing extension by $P_{i} * \operatorname{Fn}(i+1 \times \theta \times \mathbb{N}, 2)$.

\section{THE TOOLS}

Definition 2.1. AP is the set of all structures $\mathrm{a} \in H(\lambda)$, where $\mathrm{a}=\left\langle\left\{P_{i}^{\mathrm{a}}: i<\kappa\right\},\left\{\mathcal{A}_{i}^{\mathrm{a}}: i<\kappa\right\}\right\rangle$ and for each $i<\kappa$

(1) the sequence $\left\{P_{i}^{\mathrm{a}}: i<\kappa\right\}$ is a continuous $<\cdot-$ chain of ccc posets,

(2) $\mathcal{A}_{i}^{\mathrm{a}} \subset \wp\left(\mathbb{N}, P_{i+1}^{\mathrm{a}}\right)$,

(3) $P_{i+1}^{a}$ forces that the ideal generated by $\mathcal{A}_{i}^{\text {a }}$ is thin over the forcing extension by $P_{i}^{\mathrm{a}}$.

Definition 2.2. APv is the set of all structures $\mathrm{a} \in H(\lambda)$, where $\mathrm{a}=\left\langle\left\{P_{i}^{\mathrm{a}}: i<\kappa\right\},\left\{\mathcal{A}_{i}^{\mathrm{a}}: i<\kappa\right\}\right\rangle$ and for each $i<\kappa$

(1) the sequence $\left\{P_{i}^{\mathrm{a}}: i<\kappa\right\}$ is a continuous $<\cdot-$ chain of ccc posets, 
(2) $\mathcal{A}_{i}^{\mathrm{a}} \subset \wp\left(\mathbb{N}, P_{i+1}^{\mathrm{a}}\right)$,

(3) $P_{i+1}^{\mathrm{a}}$ forces that the ideal generated by $\mathcal{A}_{i}^{\mathrm{a}}$ is very thin over the forcing extension by $P_{i}^{a}$.

Definition 2.3. For $i<\kappa$, we let $\leq_{\mathrm{AP}}^{i}$ be the following two place relation on AP: $\mathrm{a} \leq_{\mathrm{AP}}^{i} \mathrm{~b}$ iff for all $j \in[i, \kappa): \mathrm{a}, \mathrm{b} \in \mathrm{AP}, P_{j}^{\mathrm{a}}<\cdot P_{j}^{\mathrm{b}}$, and $\mathcal{A}_{j}^{\mathrm{a}} \subset \mathcal{A}_{j}^{\mathrm{b}}$.

Similarly we let $\leq_{\mathrm{AP}}^{*}=\bigcup_{i<\kappa} \leq_{\mathrm{AP}}^{i}$, i.e. $\mathrm{a} \leq_{\mathrm{AP}}^{*} \mathrm{~b}$ if $\mathrm{a} \leq_{\mathrm{AP}}^{i} \mathrm{~b}$ for some $i<\kappa$.

For each a $\in \mathrm{AP}$, we may let $P_{\kappa}^{\mathrm{a}}=\bigcup\left\{P_{i}^{\mathrm{a}}: i<\kappa\right\}$ and note that $P_{i}^{\mathrm{a}}<\cdot P_{\kappa}^{\mathrm{a}}$ for all $i<\kappa$. Similarly, it follows immediately that $P_{\kappa}^{\mathrm{a}}<\cdot P_{\kappa}^{\mathrm{b}}$ whenever a $\leq_{\text {AP }}^{*} \mathrm{~b}$.

Lemma 2.4. Each of $\leq_{\mathrm{AP}}^{i}$ and $\leq_{\mathrm{AP}}^{*}$ are transitive and reflexive orders on AP. If $i<j$, then $\leq_{\mathrm{AP}}^{j} \subset \leq_{\mathrm{AP}}^{i}$. If $\mathrm{a} \leq_{\mathrm{AP}}^{i} \mathrm{~b}$ and $\mathrm{b} \leq_{\mathrm{AP}}^{i} \mathrm{a}$, then $P_{j}^{\mathrm{a}}=P_{j}^{\mathrm{b}}$ and $\mathcal{A}_{j}^{\mathrm{a}}=\mathcal{A}_{j}^{\mathrm{b}}$ for all $j \in[i, \kappa)$.

Since $A P v \subset A P$, we do not need new relation symbols to denote the same binary relations on $\mathrm{APv}$.

Lemma 2.5. If $\mathrm{a} \leq_{\mathrm{AP}}^{i} \mathrm{~b}_{1}$ for some $i<\kappa$, then there is $a \mathrm{~b}_{2} \in \mathrm{AP}$ such that $\mathrm{a} \leq_{A P}^{0} b_{2}$ and $\mathrm{b} \leq_{A P}^{i} b_{2}$. Similarly, if $\mathrm{b}_{1} \in A P v$, then $\mathrm{a} \in A P v$ and we can choose $\mathrm{b}_{2} \in \mathrm{APv}$.

Definition 2.6. For any $i<\kappa$ and ordinal $\delta$, a sequence $\left\langle\mathrm{a}_{\alpha}: \alpha<\delta\right\rangle$ is $a \leq_{\mathrm{AP}^{-}}^{i}$ increasing continuous chain if for all $\alpha<\beta<\delta$ and $j \in[i, \kappa)$ :

(1) $\mathrm{a}_{\alpha} \leq_{\mathrm{AP}}^{i} \mathrm{a}_{\beta}$,

(2) the chain $\left\{P_{j}^{\mathrm{a}_{\alpha}}: \alpha<\delta\right\}$ is strongly continuous, and,

(3) if $\alpha$ is a limit, then $\mathcal{A}_{j}^{\mathrm{a}_{\alpha}}=\bigcup\left\{\mathcal{A}_{j}^{\mathrm{a} \xi}: \xi<\alpha\right\}$.

Lemma 2.7. Suppose that $\left\{\mathrm{a}_{\alpha}: \alpha<\delta\right\}$ is a $\leq_{\mathrm{AP}}^{*}$-chain for some limit ordinal $\delta<\lambda$ and that there is a cub $C \subset \delta$ and an $i<\kappa$ such that $\left\{\mathrm{a}_{\alpha}: \alpha \in C\right\}$ is a $\leq_{\mathrm{AP}}^{i}$-increasing continuous chain. Then there is an $\mathrm{a}_{\delta} \in \mathrm{AP}$ so that

(1) $\left\{\mathrm{a}_{\alpha}: \alpha \in C \cup\{\delta\}\right\}$ is also $a \leq_{\mathrm{AP}}^{i}$-increasing continuous chain,

(2) $\mathrm{a}_{\min (C)} \leq_{\mathrm{AP}}^{0} \mathrm{a}_{\delta}$, and

(3) $\mathrm{a}_{\alpha} \leq_{\mathrm{AP}}^{*} \mathrm{a}_{\delta}$ for all $\alpha<\delta$.

If $i=0$, then $\left\{\mathrm{a}_{\alpha}: \alpha \in C\right\}$ uniquely determines $\mathrm{a}_{\delta}$.

Lemma 2.8. If $\left\{\mathrm{a}_{\alpha}: \alpha<\lambda\right\}$ is a $\leq_{\mathrm{AP}}^{*}$-increasing chain from $\mathrm{APv}$ and if $\mathcal{A}_{i}^{\mathrm{ao}} \neq \emptyset$ for all $i<\kappa$, then the ccc forcing extension by $P=\bigcup\left\{P_{\kappa}^{a_{\alpha}}: \alpha<\lambda\right\}$ satisfies that $\mathfrak{b} \leq \kappa$.

Proof. For each $i<\kappa$, choose any $\dot{a}_{i} \in \mathcal{A}_{i}^{\text {ao }}$ and let $\dot{f}_{i}$ denote the order-preserving enumeration function from $\mathbb{N}$ onto $\dot{a}_{i}$. Note that $n \leq \dot{f}_{i}(n)$ for all $n \in \mathbb{N}$. Let $\dot{g}$ be any $P$-name of an element of $\mathbb{N}^{\mathbb{N}}$. Since $P$ is ccc, we can assume that $\dot{g}$ is a countable name. Choose any $\alpha \in \lambda$ so that $\dot{g}$ is a $P_{\kappa}^{\mathrm{a}_{\alpha}}$-name. Then similarly choose $i_{0}<\kappa$ so that $\dot{g}$ is a $P_{i_{0}}^{\mathrm{a}_{\alpha}}$-name. Since $\mathrm{a}_{0} \leq_{\mathrm{AP}}^{*} \mathrm{a}_{\alpha}$ we may choose an $i>i_{0}$ so that $\mathrm{a}_{0} \leq_{\mathrm{AP}}^{i} \mathrm{a}_{\alpha}$. Now we show that no condition $p \in P$ forces that $\dot{f}_{i}<^{*} \dot{g}$. Since $P_{i+1}^{\mathrm{a}}<\cdot P$ and each of $\dot{f}_{i}$ and $\dot{g}$ are $P_{i+1}^{\mathrm{a}}$-names, it suffices to prove that if $p \in P_{i+1}^{\mathrm{a}}$ then, for any $n_{0}$ there is an extension $p^{\prime}$ of $p$ and an $n>n_{0}$ so that $p^{\prime} \Vdash_{P_{i+1}^{\mathrm{a} \alpha}}$ " $\dot{f}_{i}(n)>\dot{g}(n)$ ". Since $\dot{a}_{i} \in \mathcal{A}_{i}^{\mathrm{a} \alpha}$ and $\mathrm{a}_{\alpha} \in \mathrm{APv}$, there is a such a $p^{\prime}$ and $n$ such that $p^{\prime} \Vdash_{P_{i+1}^{a}}$ " $\dot{a}_{i} \cap[n, \dot{g}(n)]=\emptyset "$ ". There is no loss to assuming that $p^{\prime}$ decides the value of the finite set $\left\{k<n: \dot{f}_{i}(k)<n\right\}$. If this set is empty, let $m=1$, 
otherwise, let $m$ be the maximum value. Clearly $m<n$ and we now have that $p^{\prime}$ forces $\dot{f}_{i}(m+1)>\dot{g}(n)$. Since $\dot{f}_{i}$ is an increasing function, $p^{\prime} \Vdash_{P_{i}^{\mathrm{a} \alpha}} " \dot{f}_{i}(n)>\dot{g}(n)$ " as required.

Lemma 2.9. If $\left\{\mathrm{a}_{\alpha}: \alpha<\lambda\right\}$ is a $\leq_{\mathrm{AP}}^{*}$-increasing chain then the ccc forcing extension by $P=\bigcup\left\{P_{\kappa}^{a_{\alpha}}: \alpha<\lambda\right\}$ satisfies that if $\mathcal{U} \subset \wp(\mathbb{N}, P)$ is such that $\left\{i<\kappa: \mathcal{U} \cap \mathcal{A}_{i} \neq \emptyset\right\}$ has cardinality $\kappa$ for some $\alpha<\lambda$, then $\mathcal{U}$ does not have a pseudo-intersection.

Proof. Note that $P_{\kappa}^{\mathrm{a}}<\cdot P$ for all $\alpha<\lambda$. Let $\mathcal{U} \subset \wp(\mathbb{N}, P)$ and assume that $\{i<$ $\left.\kappa: \mathcal{U} \cap \mathcal{A}_{i}^{\mathrm{a}_{\alpha}} \neq \emptyset\right\}$ is cofinal in $\kappa$. Let $\dot{b}$ be any canonical $P$-name of a subset of $\mathbb{N}$. Choose $\alpha \leq \beta<\lambda$ such that $\dot{b}$ is a $P_{\kappa}^{\mathrm{a}_{\beta}}$-name. Since $P_{\beta}^{\mathrm{a}}=\bigcup\left\{P_{i}^{\mathrm{a}_{\beta}}: i<\kappa\right\}$, there is an $i_{\beta}<\kappa$ so that $\dot{b}$ is a $P_{i_{\beta}}^{\mathrm{a}_{\beta}}$-name. Choose $i<\kappa$ so that $\mathrm{a}_{\alpha} \leq_{\mathrm{AP}}^{i} \mathrm{a}_{\beta}$. Now choose any $j<\kappa$ so that $i, i_{\beta}<j$ and $\mathcal{U} \cap \mathcal{A}_{j}^{\mathrm{a}_{\alpha}}$ is not empty. Since $\mathcal{A}_{j}^{\mathrm{a}_{\alpha}} \subset \mathcal{A}_{j}^{\mathrm{a}_{\beta}}, \mathcal{A}_{j}^{\mathrm{a}_{\alpha}}$ is forced by $P_{j+1}^{\mathrm{a}_{\beta}}$ to be thin over the forcing extension by $P_{j}^{\mathrm{a}_{\beta}}$. In particular, $P_{j}^{\mathrm{a}_{\beta}}$ forces that $\dot{b}$ is not a subset of any element of $\mathcal{U} \cap \mathcal{A}_{j}^{\mathrm{a}_{\alpha}}$. Since $P_{j}^{\mathrm{a}_{\beta}}<\cdot P_{\kappa}^{\mathrm{a}_{\beta}}<\cdot P$, this is also forced by $P$.

By Proposition 1.8 we can make the following definition.

Definition 2.10. For any $\mathrm{a} \in \mathrm{AP}$ (or $\mathrm{a} \in \mathrm{APv}$ ) and ordinal $\theta<\lambda$ say that $\mathrm{b} \in \mathrm{AP}$ is the Cohen ${ }^{\theta}$-extension of a if, for each $i<\kappa$,

(1) $P_{i}^{\mathrm{b}}=P_{i}^{\mathrm{a}} * \operatorname{Fn}(i+1 \times \theta \times \mathbb{N}, 2)$

(2) $\mathcal{A}_{i}^{\mathrm{b}}=\mathcal{A}_{i}^{\mathrm{a}}$.

Lemma 2.11. If $\mathrm{a} \in \mathrm{AP}$ (respectively $\mathrm{a} \in \mathrm{APv}$ ) and $\dot{Q} \in H(\lambda)$ is a $P_{i}^{\mathrm{a}}$-name of a poset that is forced by $P_{\kappa}^{\mathrm{a}}$ to be ccc, then there is $a \mathrm{~b} \in \mathrm{AP}$ (respectively $\mathrm{b} \in \mathrm{APv}$ ) such that $\mathrm{a} \leq_{\mathrm{AP}}^{0} \mathrm{~b}$ and $P_{\kappa}^{\mathrm{b}}=P_{\kappa}^{\mathrm{a}} * \dot{Q}$.

Proof. We define b as follows. Set $\mathcal{A}_{j}^{\mathrm{b}}=\mathcal{A}_{j}^{\mathrm{a}}$ for all $j<\kappa$. For $j<i$, let $P_{j}^{\mathrm{b}}=P_{j}^{\mathrm{a}}$, and for $j \geq i$, let $P_{j}^{\mathrm{b}}=P_{j}^{\mathrm{a}} * \dot{Q}$. By Proposition 1.5, we have that $\left\{P_{j}^{\mathrm{b}}: j<\kappa\right\}$ is a continuous $<-$-chain. By assumption, $P_{j}^{\mathrm{b}} * \dot{Q}$ is ccc for all $j<\kappa$. Now we check that $\mathcal{A}_{j}^{\mathrm{b}}$ is forced by $P_{j+1}^{\mathrm{b}}$ to be thin (respectively very thin) over the forcing extension by $P_{j}^{\mathrm{b}}$. For $j<i$ this is immediate.

Now assume that $i \leq j$ and that $\left\{\dot{H}_{n}: n \in \omega\right\}$ is a sequence of $P_{j}^{\mathrm{b}}$-names that are forced to be pairwise disjoint subsets of $[\mathbb{N}]^{\ell}$ (for some $\ell \in \omega$ ) and that $\dot{g}$ is a $P_{j}^{\mathrm{b}}$-name of an element of $\mathbb{N}^{\mathbb{N}}$. Let $\dot{a}$ be any name from $\mathcal{A}_{j}^{\mathrm{a}}$. Let $(p, q)$ be any condition in $P_{j+1}^{\mathrm{a}} * \dot{Q}=P_{j+1}^{\mathrm{b}}$. We show that $\mathrm{b} \in \mathrm{AP}$ by showing that for some $n \in \omega,(p, q)$ has an extension forcing that $\dot{H}_{n} \cap \dot{a}$ is empty. We similarly show that if a $\in \operatorname{APv}$, then for some $n \in \omega,(p, q)$ has an extension forcing that $[n, \dot{g}(n)] \cap \dot{a}$ is empty.

It will be convenient to pass to the forcing extension by $P_{j+1}^{\mathrm{a}}$ so let $p \in G_{j+1}$ be a generic filter for $P_{j+1}^{\mathrm{a}}$. Let $Q$ denote the interpretation of $\dot{Q}$ by $G_{j}=G_{j+1} \cap P_{j}^{\mathrm{a}}$. We are now working in the extension $V\left[G_{j}\right]$. Recursively define a sequence $\left\{H_{n}^{\prime}: n \in\right.$ $\mathbb{N}\} \subset[\mathbb{N}]^{\ell}$ and values $\left\{m_{n}: n \in \mathbb{N}\right\} \subset \mathbb{N}$ so that, for each $n, \max \left(H_{n}^{\prime}\right)<\min \left(H_{n+1}^{\prime}\right)$, and there is a condition $q_{n} \in Q$ stronger than $q$ such that, for some $r_{n} \in G_{j}$, $\left(r_{n}, q_{n}\right) \Vdash_{P_{j}^{\mathrm{b}}}$ " $H_{n}^{\prime}=\dot{H}_{n}$ and $\dot{g}(n)=m_{n}$ ". Since $\left\{H_{n}^{\prime}: n \in \omega\right\}$ is a pairwise disjoint sequence in the forcing extension by $P_{j}^{a}$, there is a $p^{\prime} \in G_{j+1}$ (stronger than $p$ ) and 
an $n \in \omega$ such that $p^{\prime}$ forces that $H_{n}^{\prime}$ is disjoint from $a$. Since $G_{j+1}$ is a filter, we may also assume that $p^{\prime}$ is stronger than $r_{n}$. Now we have that $\left(p^{\prime}, q_{n}\right)$ is stronger than $(p, q)$ and $\left(p^{\prime}, q_{n}\right) \Vdash_{P_{j+1}^{\mathrm{b}}}$ " $\dot{H}_{n} \cap \dot{a}=\emptyset "$. Similarly, if a $\in \mathrm{APv}$, there is an $n \in \mathbb{N}$ and a $p^{\prime} \in G_{j+1}$ stronger than each of $p$ and $r_{n}$ such that $p^{\prime}$ forces that $\dot{a}$ is disjoint from $\left[n, m_{n}\right]$. This ensures that $\left(p^{\prime}, q_{n}\right) \Vdash_{P_{j+1}^{\mathrm{b}}} "[n, \dot{g}(n)] \cap \dot{a}=\emptyset "$.

Note that it follows from Lemma 2.11 that if a $\in$ AP and if $\left\{\dot{Q}_{i}: i<\kappa\right\} \in H(\lambda)$ is a sequence such that $\dot{Q}_{i}$ is a $P_{i}^{\mathrm{a}}$-name with $\left\{P_{i} * \dot{Q}_{i}: i<\kappa\right\}$ forming a continuous $<\cdot-$ sequence, then for each $i<\kappa, P_{i+1} * \dot{Q}_{i}$ forces that $\mathcal{A}_{i}^{\text {a }}$ is thin over the extension by $P_{i} * \dot{Q}_{i}$. This means that it is only the behavior of $\dot{Q}_{i+1}$ that affects if there is $\mathrm{b} \in \mathrm{AP}$ with $\mathrm{a} \leq_{\mathrm{AP}}^{0} \mathrm{~b}$ and $P_{i}^{\mathrm{b}}=P_{i}^{\mathrm{a}} * \dot{Q}_{i}$ for all $i<\kappa$.

Definition 2.12. If a $\in \mathrm{AP}$ (respectively $\mathrm{a} \in \mathrm{APv}$ ) and $\dot{Q} \in H(\lambda)$ is a $P_{\kappa}^{\mathrm{a}}$-name such that

(1) $\dot{Q}$ has cardinality less than $\kappa$,

(2) $P_{\kappa}^{\mathrm{a}}$ forces that $\dot{Q}$ is ccc

then $\mathrm{a} * \dot{Q}$ denotes the $\leq_{\mathrm{AP}}^{0}$-extension $\mathrm{b}$ as in Lemma 2.11 where $i<\kappa$ is chosen to be minimal such that $\dot{Q}$ is a $P_{i}^{\mathrm{a}}$-name.

This next lemma illustrates the device we use to ensure that every ultrafilter will have pseudo-intersection number at most $\kappa$.

Lemma 2.13. Suppose that $\delta<\lambda$ has cofinality $\kappa$ and that $\left\{\mathrm{a}_{\alpha}: \alpha \in \delta\right\} \subset \mathrm{AP}$ is $a \leq_{\mathrm{AP}}^{*}$-increasing sequence. Further suppose that there is a cub $C \subset \delta$ of order type

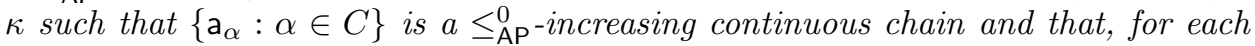
$\alpha \in \operatorname{acc}(C), \mathrm{a}_{\alpha+1}$ is a Cohen ${ }^{\omega_{1}}$-extension of $\mathrm{a}_{\alpha}$. Then, if $P=\bigcup\left\{P_{\kappa}^{\mathrm{a}_{\alpha}}: \alpha \in \operatorname{acc}(C)\right\}$ and $\mathcal{E} \subset \wp(\mathbb{N}, P)$ is a maximal family that is forced to be a free ultrafilter on $\mathbb{N}$, there is $a \mathrm{~b} \in \mathrm{AP}$ such that $\mathrm{a}_{\alpha} \leq_{\mathrm{AP}}^{*} \mathrm{~b}$ for all $\alpha \in \delta, P=P_{\kappa}^{\mathrm{b}}$, and, for all $i<\kappa$, $\mathcal{E} \cap \mathcal{A}_{i}^{\mathrm{b}}$ is not empty.

Proof. Let $\mathcal{E} \subset \wp(\mathbb{N}, P)$ and assume that $1_{P}$ forces that $\mathcal{E}$ is a free ultrafilter on $\mathbb{N}$ and that $\mathcal{E}$ is a maximal such family. This just means that if $\dot{b} \in \wp(\mathbb{N}, P)$ and $1_{p} \Vdash_{P} "(\exists \dot{e} \in \mathcal{E}) \dot{b} \supset \dot{e} "$, then $\dot{b} \in \mathcal{E}$. Let $\left\{\alpha_{i}: i<\kappa\right\}$ be the order-preserving enumeration of $\operatorname{acc}(C)$. For each $i<\kappa$ we now describe how to choose a value $\beta_{i} \in \omega_{1}$. By our assumption, a $\alpha_{\alpha_{i}+1}$ is a Cohen ${ }^{\omega_{1}}$-extension. That is, $P_{i}^{\mathrm{a}_{\alpha_{i}+1}}$ is equal to $P_{i+1}^{\mathrm{a}_{\alpha_{i}} * \dot{Q}_{i+1}^{\mathrm{a} \alpha_{i}}}$ where $\dot{Q}_{i+1}^{\mathrm{a}_{\alpha_{i}}}$ is equal to (the trivial) $P_{i+1}^{\mathrm{a}_{\alpha_{i}}}$-name for $\operatorname{Fn}\left(i+1 \times \omega_{1} \times \mathbb{N}, 2\right)$. Let $\vec{x}_{i}$ denote the canonical $\omega_{1}$-sequence associated with $\operatorname{Fn}\left(\{i\} \times \omega_{1} \times \mathbb{N}, 2\right)$ for this particular copy of Cohen forcing. Similarly, let $\left\{c\left(\vec{x}_{i}, \beta\right), a\left(\vec{x}_{i}, \beta\right): \beta<\omega_{1}\right\} \subset$ $\wp\left(\mathbb{N}, P_{i+1}^{\mathrm{a}_{\alpha_{i}+1}}\right)$ be the family of names as constructed as in Definition 1.1. Since the family $\left\{c\left(\vec{x}_{i}, \beta\right): \beta<\omega_{1}\right\}$ is forced to be pairwise almost disjoint, there is a maximal antichain $A_{i} \subset P$ such that for each $p \in A_{i}$, there is a $\beta_{p}$ such that $p$ forces that $a\left(\vec{x}_{i}, \xi\right)$ is in $\mathcal{E}$ for all $\beta_{p}<\xi \in \omega_{1}$. Since $P$ is ccc, $A_{i}$ is countable, and so we may choose any value $\beta_{i} \in \omega_{1}$ that is larger than $\beta_{p}$ for each $p \in A_{i}$. It follows that $1 \Vdash_{P}$ " $(\exists \dot{e} \in \mathcal{E}) a\left(\vec{x}_{i}, \beta_{i}\right) \supset \dot{e} "$. By the maximality assumption on $\mathcal{E}, a\left(\vec{x}_{i}, \beta_{i}\right) \in \mathcal{E}$.

Now we define b. For each $i<\kappa, P_{i}^{\mathrm{b}}=P_{i}^{\mathrm{a}_{\alpha_{i}}}$ and $\mathcal{A}_{i}^{\mathrm{b}}=\mathcal{A}_{i}^{\mathrm{a}_{\alpha_{i}}} \cup\left\{a\left(\vec{x}_{i}, \beta_{i}\right)\right\}$. Evidently we have that $\mathcal{E} \cap \mathcal{A}_{i}^{\mathrm{b}}$ is not empty for all $i<\kappa$. Since $P_{i+1}^{\mathrm{a}_{\alpha_{i}+1}}<\cdot P_{i+1}^{\mathrm{a}_{\alpha_{i+1}}}$, we have that $\mathcal{A}_{i}^{\mathrm{b}}$ is a subset of $\wp\left(\mathbb{N}, P_{i+1}^{\mathrm{b}}\right)$. It follows from Proposition 1.6 that $\mathcal{A}_{i}^{\mathrm{b}}$ is forced to be thin over the forcing extension by $P_{i}^{\mathrm{b}}=P_{i}^{\mathrm{a}_{\alpha_{i}}}$. Now for $i<j<\kappa$, $P_{i}^{\mathrm{b}}=P_{i}^{\mathrm{a}_{\alpha_{i}}}<\cdot P_{j}^{\mathrm{a}_{\alpha_{i}}}<\cdot P_{j}^{\alpha_{j}}=P_{j}^{\mathrm{b}}$. Now suppose that $j<\kappa$ is a limit of uncountable 
cofinality, we have to check that $P_{j}^{\mathrm{b}}=\bigcup\left\{P_{i}^{\mathrm{b}}: i<j\right\}$. Let $p \in P_{j}^{\mathrm{b}}$. Since $P_{j}^{\mathrm{b}}=$ $P_{j}^{\mathrm{a}_{\alpha_{j}}}=\bigcup\left\{P_{i}^{\mathrm{a}_{\alpha_{j}}}: i<j\right\}$, we may choose $i_{1}<j$ such that $p \in P_{i_{1}}^{\mathrm{a}_{\alpha_{j}}}$. By the assumption that $\left\{\mathrm{a}_{\alpha}: \alpha \in C\right\}$ is a $\leq_{\mathrm{AP}}^{0}$-increasing continuous chain, there is an $i<j$ with $i_{1} \leq i$ and $p \in P_{i_{1}}^{\mathrm{a}_{\alpha_{i}}}$. Finally, $p \in P_{i_{1}}^{\mathrm{a}_{\alpha_{i}}} \subset P_{i}^{\mathrm{a}_{\alpha_{i}}}=P_{i}^{\mathrm{b}}$ which completes this step. It also shows that $P_{\kappa}^{\mathrm{b}}=P$. This completes the verification that $\mathrm{b} \in \mathrm{AP}$. Fix any $\xi<\delta$ we verify that $\mathrm{a}_{\xi} \leq_{\text {AP }}^{*} b$. Choose $i<\kappa$ so that $\xi<\alpha_{i}$ and choose $i^{*}<\kappa$ so that $\mathrm{a}_{\xi} \leq_{\mathrm{AP}}^{i^{*}} \mathrm{a}_{\alpha_{i}}$. We show that $\mathrm{a}_{\xi} \leq_{\mathrm{AP}}^{i^{*}}$ b. Let $i^{*} \leq j<\kappa$. First we have that $\mathcal{A}_{j}^{\mathrm{a} \xi} \subset \mathcal{A}_{j}^{\alpha_{i}} \subset A_{j}^{\alpha_{j}} \subset A_{j}^{\mathrm{b}}$. Secondly, $P_{j}^{\mathrm{a} \xi}<\cdot P_{j}^{\mathrm{a}_{\alpha_{i}}}<\cdot P_{j}^{\mathrm{a}_{\alpha_{j}}}=P_{j}^{\mathrm{b}}$.

The proof of this next lemma is the same so the proof is omitted.

Lemma 2.14. Suppose that $\left\{\mathrm{a}_{\alpha}: \alpha \in \kappa\right\} \subset \mathrm{APv}$ is a $\leq_{\mathrm{AP}}^{0}$-increasing continuous chain for some $i<\kappa$ and that, for each limit $\alpha \in \kappa, \mathrm{a}_{\alpha+1}$ is a Cohen ${ }^{\omega_{1}}$-extension of $\mathrm{a}_{\alpha}$. Then, if $P=\bigcup\left\{P_{\kappa}^{\mathrm{a}_{\alpha}}: \alpha<\kappa\right\}$ and $\mathcal{E} \subset \wp(\mathbb{N}, P)$ is a maximal family that is forced to be a free ultrafilter on $\mathbb{N}$, there is a $\mathrm{b} \in \mathrm{APv}$ such that $\mathrm{a}_{\alpha} \leq_{\mathrm{AP}}^{*} \mathrm{~b}$ for all $\alpha \in \kappa, P=P_{\kappa}^{\mathrm{b}}$, and $\left\{i<\kappa: \mathcal{E} \cap \mathcal{A}_{i}^{\mathrm{b}} \neq \emptyset\right\}$ has cardinality $\kappa$.

\section{THE LAVER STYLE POSETS}

In this section we develop the tools to allow us incorporate posets into $\leq_{\mathrm{AP}}^{*}$ chains that will increase the splitting number. An ultrafilter $\mathcal{D}$ on $\mathbb{N}$ is Ramsey if for each function $f$ with domain $\mathbb{N}$ and range an ordinal, there is a $D \in \mathcal{D}$ such that $f \uparrow D$ is either constant or is strictly increasing. For any family $\mathcal{D}$ of subsets of $\mathbb{N}$ that has the finite intersection property, we let $\langle\mathcal{D}\rangle$ denote the filter generated by $\mathcal{D}$. We use the standard notation, $\mathcal{D}^{+}$, to denote the set of subsets of $\mathbb{N}$ that meet every member of $\mathcal{D}$.

Proposition 3.1. If $\mathcal{D}_{0}$ is a free filter on $\mathbb{N}$ and $\theta$ is an ordinal with $\theta \geq \mathfrak{c}$, then $\mathcal{D}_{0}$ can be extended to a Ramsey ultrafilter in the forcing extension by $\operatorname{Fn}(\theta, 2)$.

Definition 3.2. For a filter $\mathcal{D}$ on $\omega$, we define the Laver style poset $\mathbb{L}(\mathcal{D})$ to be the set of trees $T \subset \mathbb{N}^{<\omega}$ with the property that $T$ has a minimal branching node

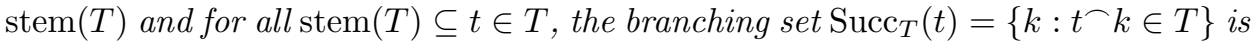
an element of $\mathcal{D}$. For any tree $T \subset \mathbb{N}^{<\omega}$ and $t \in T$, we let $T_{t}=\{s \in T: s \cup t \in T\}$.

The name $\dot{L}_{\mathcal{D}}=\left\{(k, T):(\exists t) T=\left(\mathbb{N}^{<\omega}\right)_{t-k}\right\}$ will be referred to as the canonical name for the real (pseudo-intersection) added by $\mathbb{L}(\mathcal{D})$.

Proposition 3.3. If $\mathcal{D}$ is any free filter on $\mathbb{N}$, then $\dot{L}_{\mathcal{D}}$ is forced to be a pseudointersection for $\mathcal{D}$ and for every function $f \in \mathbb{N}^{\mathbb{N}}$, the enumeration function of $\dot{L}_{\mathcal{D}}$ is forced to be mod finite greater than $f$.

Definition 3.4. If $E$ is a dense subset of $\mathbb{L}(\mathcal{D})$, then there is a (rank) function $\rho_{E}$ from $\mathbb{N}^{<\omega}$ into $\omega_{1}$ where $\rho_{E}(t)=0$ if and only if $t=\operatorname{stem}(T)$ for some $T \in E$, and for all $t \in \mathbb{N}<\omega$ and $0<\alpha \in \omega_{1}, \rho_{E}(t)=\alpha$ if $\alpha$ is minimal such that the set $\left\{k \in \omega: \rho_{E}(t \frown k)<\alpha\right\}$ is in $\mathcal{D}^{+}$.

Proposition 3.5. If $\mathcal{D}$ is a Ramsey ultrafilter and $E \subset \mathbb{L}(\mathcal{D})$ is a dense set, then for each $t \in \mathbb{N}<\omega$ with $\rho_{E}(t)>0$, there is a $D_{t} \in \mathcal{D}$ such that $\left\{\rho_{E}(t \frown k): k \in D_{t}\right\}$ is increasing and cofinal in $\rho_{E}(t)$.

Lemma 3.6 $([10,1.9])$. Suppose that $\mathbb{P}, \mathbb{Q}$ are posets with $\mathbb{P}<\cdot \mathbb{Q}$. Suppose also that $\dot{\mathcal{D}}_{0}$ is a $\mathbb{P}$-name of a filter on $\mathbb{N}$ and $\dot{\mathcal{D}}_{1}$ is a $\mathbb{Q}$-name of a filter on $\mathbb{N}$. If $\Vdash_{\mathbb{Q}} \dot{\mathcal{D}}_{0} \subseteq \dot{\mathcal{D}}_{1}$ 
then $\mathbb{P} * \mathbb{L}\left(\dot{\mathcal{D}}_{0}\right)$ is a complete subposet of $\mathbb{Q} * \mathbb{L}\left(\dot{\mathcal{D}}_{1}\right)$ if either of the two equivalent conditions hold:

(1) $\Vdash_{\mathbb{Q}}\left(\wp(\mathbb{N}, \mathbb{P}) \cap \dot{\mathcal{D}}_{0}^{+}\right) \subseteq \dot{\mathcal{D}}_{1}^{+}$,

(2) $\Vdash_{\mathbb{Q}} \dot{\mathcal{D}}_{1} \cap \wp(\mathbb{N}, \mathbb{P}) \subseteq\left\langle\dot{\mathcal{D}}_{0}\right\rangle$.

Proof. Let $\dot{E}$ be any $\mathbb{P}$-name of a maximal antichain of $\mathbb{L}\left(\dot{\mathcal{D}}_{0}\right)$. By Lemma 1.5, it suffices to show that $\mathbb{Q}$ forces that every member of $\mathbb{L}\left(\dot{\mathcal{D}}_{1}\right)$ is compatible with some member of $\dot{E}$. Let $G$ be any $\mathbb{Q}$-generic filter and let $E$ denote the valuation of $\dot{E}$ by $G \cap \mathbb{P}$. Working in the model $V[G \cap \mathbb{P}]$, we have the function $\rho_{E}$ as in Lemma 3.4] Choose $\delta \in \omega_{1}$ satisfying that $\rho_{E}(t)<\delta$ for all $t \in \omega^{<\omega}$. Now, working in $V[G]$, we consider any $T \in \mathbb{L}\left(\dot{\mathcal{D}}_{1}\right)$ and we find an element of $E$ that is compatible with $T$. In fact, by induction on $\alpha<\delta$, one easily proves that for each $T \in \mathbb{L}\left(\dot{\mathcal{D}}_{1}\right)$ with $\rho_{E}(\operatorname{stem}(T)) \leq \alpha, T$ is compatible with some member of $E$.

If $\dot{\mathcal{D}_{0}}$ is the $\mathbb{P}$-name of a maximal filter (ultrafilter), then the conditions in Lemma 3.6 hold.

Lemma 3.7. If $V \subset V^{\prime}$ are models and $\mathcal{A} \in V^{\prime}$ is thin over $V$, then for every Ramsey ultrafilter $\mathcal{D} \in V$, there is an ultrafilter $\mathcal{D}^{\prime} \supset \mathcal{D}$ in $V^{\prime}$ such that, for each $V^{\prime}$-generic filter $G^{\prime}$ for $\mathbb{L}\left(\mathcal{D}^{\prime}\right), \mathcal{A}$ is thin over $V\left[G^{\prime} \cap \mathbb{L}(\mathcal{D})\right]$. In other words, in the forcing extension of $V^{\prime}$ by $\mathbb{L}\left(\mathcal{D}^{\prime}\right), \mathcal{A}$ is thin over the forcing extension of $V$ by $\mathbb{L}(\mathcal{D})$.

Proof. Let $\mathcal{O}$ denote the set of strictly increasing functions $f \in V$ such that $f \in \mathbb{N}^{D}$ for some $D \in \mathcal{D}$. By the definition of thin over $V$, we may assume that $\mathcal{A}$ is closed under finite unions. For each $D \in \mathcal{D}, a \in \mathcal{A}$ and $f \in \mathcal{O}$, let $E(D, f, a)=\{n \in$ $D \cap \operatorname{dom}(f): f(n) \notin a\}$. We show that the family $\{E(D, f, a): f \in \mathcal{O}, D \in \mathcal{D}, a \in$ $\mathcal{A}\}$ has the finite intersection property. It suffices to prove that if $\left\{f_{k}: k<\ell\right\}$ is a finite subset of $\mathcal{O}, D \in \mathcal{D}$, and $a \in \mathcal{A}$, then there is an $n \in D$ such that $f_{k}(n) \notin a$ for all $k<\ell$. By shrinking $D$ we can assume that $D \subset \operatorname{dom}\left(f_{k}\right)$ for each $k<\ell$. Choose any stictly increasing function $f \in V$ satisfying that for all $n \in \mathbb{N}$, $[f(n), f(n+1)) \cap D \neq \emptyset$, and for all $j \in D$ with $j \leq f(n), f_{k}(j)<f(n+1)$ for each $k<\ell$. Therefore, for each $n \in \mathbb{N}$ and $j \in D \cap[f(n), f(n+1))$, we have that $f(n-1)<f_{k}(j)<f(n+1)$ for all $k<\ell$. By re-indexing, we can assume that $\bigcup\{[f(3 n), f(3 n+1)): n \in \mathbb{N}\}$ is in $\mathcal{D}$. Since $\mathcal{D}$ is Ramsey, we may choose $D_{1} \subset D$ so that $D_{1}=\left\{j_{n}: n \in \mathbb{N}\right\}$ and $f(3 n) \leq j_{n}<f(3 n+1)$ for all $n \in \mathbb{N}$. Now define $H_{n}=\left\{f_{k}\left(j_{n}\right): k<\ell\right\}$ and observe that $H_{n} \subset[f(3 n-1), f(3 n+2))$ and so the sequence $\left\{H_{n}: n \in \mathbb{N}\right\}$ consists of pairwise disjoint sets. Since $\mathcal{A}$ is thin over $V$, there is an $n$ such that $H_{n} \cap a$ is empty. It follows that $j_{n} \in D$ and $f_{k}\left(j_{n}\right) \notin a$ for each $k<\ell$ as required.

Let $\mathcal{D}^{\prime}$ be any ultrafilter in $V^{\prime}$ extending the family $\{E(D, f, a): f \in \mathcal{O}, D \in$ $\mathcal{D}, a \in \mathcal{A}\}$. Now we let $\left\{\dot{H}_{n}: n \in \omega\right\}$ be a sequence in $V$ of $\mathbb{L}(\mathcal{D})$-names that are forced by some $T_{0} \in \mathbb{L}(\mathcal{D})$ to be pairwise disjoint and of cardinality at most $\ell \in \omega$. Let $a$ be any element of $\mathcal{A}$ and $T_{0}^{\prime} \in \mathbb{L}\left(\mathcal{D}^{\prime}\right)$ be any condition stronger than $T_{0}$. We prove there is an extension $T_{0}^{\prime} \supset T_{1}^{\prime} \in \mathbb{L}\left(\mathcal{D}^{\prime}\right)$ and an $n \in \omega$ such that $T_{1}^{\prime} \Vdash \dot{H}_{n} \cap a$ is empty. Let $t_{0}=\operatorname{stem}\left(T_{0}\right)$ and for each $1<n \in \omega$, let $H_{n, 0}$ be the maximal set such that there is a $T_{n} \in \mathbb{L}(\mathcal{D})$ with $T_{n} \Vdash H_{n, 0} \subset \dot{H}_{n}$ and $\operatorname{stem}\left(T_{n}\right)=t_{0}$. There is a $D_{0} \in \mathcal{D}$ so that each element of the sequence $\left\{H_{n, 0}: n \in D_{0}\right\}$ has the same cardinality. Since we can assume that $D_{0} \subset \operatorname{Succ}_{T_{0}}\left(t_{0}\right)$, it follows that the elements of $\left\{H_{n, 0}: n \in D_{0}\right\}$ are pairwise disjoint. Choose any $1<n \in D_{0}$ so that $H_{n, 0} \cap a$ 
is empty. If $T_{n} \Vdash \dot{H}_{n}=H_{n, 0}$, then we are done because $T_{n}$ and $T_{0}^{\prime}$ have the same stem, and so are compatible. Let $\ell^{\prime} \leq \ell$ be the value such that $T_{n} \Vdash\left|\dot{H}_{n} \backslash H_{n, 0}\right|=\ell^{\prime}$ and let $E_{0}=\left\{T \in \mathbb{L}(\mathcal{D}): \operatorname{stem}(T) \notin T_{n}\right.$ or $\left.(\exists j) T \Vdash j \in \dot{H}_{n} \backslash H_{n, 0}\right\}$. Since $E_{0}$ is a dense subset of $\mathbb{L}(\mathcal{D})$, we have the associated rank function $\rho_{E_{0}}$ where for $t \in T_{n}$, $\rho_{E_{0}}(t)=0$ implies that there is a $T \in E_{0}$ with $\operatorname{stem}(T)=t$ and $j \in \mathbb{N} \backslash H_{n, 0}$ such that $T \Vdash j \in \dot{H}_{n}$. By the maximality assumption on $T_{n}$, we have that $\rho_{E_{0}}\left(t_{0}\right)>0$. If $\rho_{E_{0}}\left(t_{0}\right)>1$, then by Proposition 3.5. there is a $k_{0}$ such that $1 \leq \rho_{E_{0}}\left(t_{0} k_{0}\right)<\rho_{E_{0}}\left(t_{0}\right)$ and $t_{0} k_{0} \in T_{0}^{\prime}$. By repeating this step finitely many times, we can find a $t_{1} \in T_{0}^{\prime}$ such that $\rho_{E_{0}}\left(t_{1}\right)$ is equal to 1 . We may assume that $\rho_{E_{0}}\left(t_{1} k\right)=0$ for all $k \in \operatorname{Succ}_{T_{0}}\left(t_{1}\right)$. For each $k \in \operatorname{Succ}_{T_{0}}\left(t_{1}\right)$, let $H_{n}\left(t_{1} k\right)$ be the maximal (non-empty) set of $j$ such that there is some condition in $\mathbb{L}(\mathcal{D})$ with stem equal to $t_{1} k$ that forces $H_{n}\left(t_{1} k\right) \subset \dot{H}_{n} \backslash H_{n, 0}$. There is some $D_{t_{1}} \in \mathcal{D}$ and $\ell_{t} \in \omega$ such that $\left\{H_{n}\left(t_{1} k\right): k \in D_{t_{1}}\right\}$ all have cardinality $\ell_{t}$. For each $j<\ell_{t}$, define the function $f_{j}$ with domain $D_{t_{1}}$ such that $f_{j}(k)$ is in $H_{n}\left(t_{1} k\right)$ and, for each $k \in D_{t_{1}},\left\{f_{j}(k): j<\ell_{t}\right\}$ enumerates $H_{n}\left(t_{1} k\right)$ in increasing order. By shrinking $D_{t_{1}}$ we can assume that each $f_{j} \uparrow D_{t_{1}}$ is either constant or is strictly increasing. Since $\rho_{E_{0}}\left(t_{1}\right)>0, f_{0}$ is not constant. To see this, assume that $f_{0}(k)=m$ for each $k \in D_{t_{1}}$. For each $k \in D_{t_{1}}$, choose a condition $T^{k} \in \mathbb{L}(\mathcal{D})$ so that $\operatorname{stem}\left(T^{k}\right)=t_{1} k$ and $T^{k} \Vdash m \in \dot{H}_{n}$. But now, the contradiction is that $\bigcup\left\{T^{k}: k \in D_{t_{1}}\right\}$ can be shown to be a condition with stem equal to $t_{1}$ that forces that $m \in \dot{H}_{n}$. Choose any $k$ in the non-empty set $\operatorname{Succ}_{T_{0}^{\prime}}\left(t_{1}\right) \cap \bigcap\left\{E\left(D_{t_{1}}, f_{j}, a\right): j<\ell^{\prime}\right\}$ and set $t_{2}=t_{1} k \in T_{0}^{\prime}$. Now define $H_{n, 1}=H_{n, 0} \cup H_{n}\left(t_{1} k\right)$ and choose $T_{2} \subset T_{0}$ as above so that $\operatorname{stem}\left(T_{2}\right)=t_{2}$ and $T_{2} \Vdash H_{n, 1} \subset \dot{H}_{n}$. Define $E_{2}$ analogous to how we defined $E_{0}$ so that for $t \in\left(T^{k}\right)_{t_{2}}, \rho_{E_{2}}(t)=0$ if and only if there is a condition with stem $t$ that forces some $j$ to be in $\dot{H}_{n} \backslash H_{n, 1}$. We again note that when we proved that each $f_{j}\left(j<\ell^{\prime}\right)$ above was strictly increasing, we have also shown that $\rho_{E_{2}}\left(t_{2}\right)>0$. Since $H_{n, 1}$ is a proper extension of $H_{n, 0}$ and is also disjoint from $a$, we can repeat this argument finitely many (at most $\ell$ ) times until we have found an element $t \in T_{0}^{\prime}$ which has a stem preserving extension that forces $\dot{H}_{n}$ is disjoint from $a$.

Corollary 3.8. Suppose that $\mathrm{a} \in \mathrm{AP}$ and let $\left|P_{\kappa}^{\mathrm{a}}\right| \leq \theta=\theta^{\aleph_{0}}<\lambda$, then there is a $\mathrm{b} \in \mathrm{AP}$ and a sequence $\left\{\mathcal{D}_{i}: i<\kappa\right\}$ satisfy that, for $i<j<\kappa$,

(1) $\mathrm{a} \leq_{\mathrm{AP}}^{0} \mathrm{~b}$,

(2) $\mathcal{D}_{i} \subset \wp\left(\mathbb{N}, P_{i} * \operatorname{Fn}(i+1 \times \theta, 2)\right)$,

(3) $\mathcal{D}_{i} \subset \mathcal{D}_{j}$,

(4) $P_{i} * \operatorname{Fn}(i+1 \times \theta, 2)$ forces that $\mathcal{D}_{i}$ is a Ramsey ultrafilter on $\mathbb{N}$,

(5) $P_{i}^{\mathrm{b}}=P_{i}^{\mathrm{a}} * \operatorname{Fn}(i \times \theta, 2) * \mathbb{L}\left(\mathcal{D}_{i}\right)$.

Definition 3.9. For any $\mathrm{a} \in \mathrm{AP}$ we will say that $\mathrm{b}$ is an $\mathbb{L}(\overrightarrow{\mathcal{D}})$-extension of $\mathrm{a}$ if there is a sequence $\left\{\mathcal{D}_{i}: i<\kappa\right\}$ such that for all $i<j<\kappa$,

(1) $a \leq_{A P}^{0} b$,

(2) $\mathcal{D}_{i} \subset \wp\left(\mathbb{N}, P_{i}^{\mathrm{a}}\right)$,

(3) $\mathcal{D}_{i}$ is forced by $P_{i}^{a}$ to be a Ramsey ultrafilter on $\mathbb{N}$,

(4) $\mathcal{D}_{i} \subset \mathcal{D}_{j}$,

(5) $P_{i}^{\mathrm{b}}=P_{i}^{\mathrm{a}} * \mathbb{L}\left(\mathcal{D}_{i}\right)$. 
4. $\pi p(\mathfrak{U}) \leq \kappa$ AND $\mathfrak{b}=\mathfrak{s}=\lambda$

Fix a 1-to-1 function $h$ from $\lambda$ onto $H(\lambda)$. Recall that $\left\{X_{\alpha}: \alpha \in E\right\}$ is the $\diamond$-sequence on $\lambda$ as in $\operatorname{Hyp}(\kappa, \lambda)$.

Theorem 4.1. Assume $\operatorname{Hyp}(\kappa, \lambda)$. There is a sequence $\left\{\mathrm{a}_{\alpha}, \zeta_{\alpha}: \alpha \in \lambda\right\}$ such that for each limit $\delta \in \lambda$

(1) the sequence $\left\{\mathrm{a}_{\alpha}: \alpha<\delta\right\}$ is $\leq_{\mathrm{AP}}^{*}$-increasing, $\left\{\zeta_{\alpha}: \alpha<\delta\right\}$ is non-decreasing, and $\zeta_{\delta} \in \lambda$ is the supremum of $\left\{\zeta_{\alpha}: \alpha<\delta\right\}$,

(2) if $\delta \notin E$, the sequence $\left\{\mathrm{a}_{\alpha}: \alpha \in \operatorname{acc}\left(C_{\delta}\right) \cup\{\delta\}\right\}$ is a $\leq_{\mathrm{AP}}^{0}$-increasing continuous chain,

(3) if $\delta \in E$ and $\mathcal{E}_{\delta}=\left\{h(\xi): \xi \in X_{\delta}\right\}$ is a maximal subset of $\wp\left(\mathbb{N}, P_{\kappa}^{\mathbf{a}_{\delta}}\right)$ that is forced by $P_{\kappa}^{\mathrm{a} \delta}$ to be a free ultrafilter on $\mathbb{N}$, then $\mathcal{E}_{\delta} \cap \mathcal{A}_{i}^{\mathrm{a}_{\delta}}$ is not empty for all $i<\kappa$,

(4) $\mathrm{a}_{\delta+1}$ is the Cohen ${ }^{\omega_{1}}$-extension of $\mathrm{a}_{\delta}$ and $\zeta_{\delta+1}=\zeta_{\delta}$,

(5) if $\alpha=\delta+1$ then $\zeta_{\alpha+1}=\zeta_{\alpha}$ and $\mathrm{a}_{\alpha+1}$ is the Cohen ${ }^{\theta_{\alpha}}$-extension of $\mathrm{a}_{\alpha}$ where $\theta_{\alpha}=\left|P_{\kappa}^{\mathrm{a}_{\alpha}}\right|^{\aleph_{0}}$

(6) if $\alpha=\delta+2$, then $\zeta_{\alpha+1}=\zeta_{\alpha}$ and $\mathrm{a}_{\alpha+1}$ is an $\mathbb{L}(\mathcal{D})$-extension of $\mathrm{a}_{\alpha}$,

(7) if $\alpha \in(\delta+2, \delta+\omega)$, then $\zeta_{\alpha+1}$ is the minimal value strictly above $\zeta_{\alpha}$ such that $\dot{Q}_{\alpha+1}=h\left(\zeta_{\alpha+1}-1\right)$ has cardinality less than $\kappa$ and is a $P_{\kappa}^{\mathrm{a}_{\alpha}}$-name of a poset that is forced to be ccc, and $\mathrm{a}_{\alpha+1}=\mathrm{a}_{\alpha} * \dot{Q}_{\alpha+1}$ as in Definition 2.12.

Proof. The proof is by induction on limit $\delta<\lambda$. We can define $\mathrm{a}_{0}$ so that $P_{i}^{\mathrm{a}_{0}}=$ $\operatorname{Fn}(i \times \mathbb{N}, 2)$ and $\mathcal{A}_{i}^{\text {a }}=\emptyset$ for all $i<\kappa$. Similarly, for $n \in \omega$, let $\mathrm{a}_{n+1}$ be the Cohen ${ }^{\omega}$-extension of $\mathrm{a}_{n}$. For all $n \in \omega$, set $\zeta_{n}=0$. If $\delta$ is a limit ordinal not in $E$ and $\operatorname{acc}\left(C_{\delta}\right)$ is cofinal in $\delta$, then $\left\{\mathrm{a}_{\alpha}: \alpha \in \operatorname{acc}\left(C_{\delta}\right)\right\}$ is a $\leq_{A \mathrm{P}}^{0}$-increasing continuous chain, and so $\mathrm{a}_{\delta}$ is defined as in Proposition 2.7. If $\operatorname{acc}\left(C_{\delta}\right)$ is not cofinal in $\delta$, then let $\alpha_{0}$ be the maximum element of $\operatorname{acc}\left(C_{\delta}\right)$, and let $C=\left\{\alpha_{n}: n \in \omega\right\}$ enumerate $C_{\delta} \backslash \alpha_{0}$. There is an $i<\kappa$ such that $\left\{\mathrm{a}_{\alpha_{n}}: n \in \omega\right\}$ is a $\leq_{A \mathrm{AP}}^{i}$-increasing continuous chain. Again applying Proposition 2.7 produces $\mathrm{a}_{\delta}$ so that $\mathrm{a}_{\alpha_{0}} \leq_{\mathrm{AP}}^{0} \mathrm{a}_{\delta}$. Case (5) is handled by Proposition 1.8 per Definition 2.10. Similarly, per Definition 3.9 and by inductive assumption (5), Corollary 3.8 handles inductive step (6). Inductive step (7) is handled by Definition 2.12 and Lemma 2.11

Now we consider inductive step (3) when $\delta \in E$. By induction hypothesis (1), we have that $\left\{\mathrm{a}_{\alpha}: \alpha \in \operatorname{acc}\left(C_{\delta}\right)\right\}$ is a $\leq_{A \mathrm{P}}^{0}$-increasing chain. Let $P$ denote the poset $\bigcup\left\{P_{\kappa}^{\mathrm{a}_{\alpha}}: \alpha \in \operatorname{acc}\left(C_{\delta}\right)\right\}$. We recall from Lemma 2.9 that $P_{\kappa}^{\mathrm{a} \beta}<\cdot P$ for all $\beta<\delta$. If $\left\{h(\xi): \xi \in X_{\delta}\right\}$ is a subset of $\wp(\mathbb{N}, P)$ and is forced to have the finite intersection property, we can use Zorn's Lemma to enlarge it to a maximal such family. Otherwise, choose $\mathcal{E}_{\delta} \subset \wp(\mathbb{N}, P)$ to be any maximal family which is forced to have the finite intersection property. Since $\wp(\mathbb{N}, P)$ consists only of sets that are forced to be infinite, $\mathcal{E}_{\delta}$ is forced to be a free filter. We prove that $\mathcal{E}_{\delta}$ is forced to be an ultrafilter. Assume that $\dot{b}$ is any canonical $P$-name of a subset of $\mathbb{N}$ and that $p \in P$ is any condition that forces $\dot{b}$ meets every member of $\mathcal{E}_{\delta}$. There is a $\dot{e} \in \wp(\mathbb{N}, P)$ such that $p \Vdash_{P} " \dot{b}=\dot{e} "$ and, for all $q \in P$ that are incomparable with $p, q \Vdash_{P}$ " $\dot{e}=\mathbb{N}$ ". Clearly then $1_{P}$ forces that $\mathcal{E}_{\delta} \cup\{\dot{e}\}$ has the finite intersection property. By the maximality of $\mathcal{E}_{\delta}, \dot{e} \in \mathcal{E}_{\delta}$. This proves that $p \Vdash \dot{b} \in \mathcal{E}_{\delta}$. Now apply Lemma 2.13.

This completes the proof. 
Theorem 4.2. Assume $\operatorname{Hyp}(\kappa, \lambda)$. There is a ccc poset $P$ forcing that $\mathfrak{s}=\mathfrak{b}=\lambda$, $M A(\kappa)$, and $\pi p(\mathcal{U}) \leq \kappa$ for all free ultrafilters $\mathcal{U}$ on $\mathbb{N}$.

Proof. Let $\left\{\mathrm{a}_{\alpha}, \zeta_{\alpha}: \alpha \in \lambda\right\}$ be the sequence constructed in Theorem 4.1, Let $P$ be the poset $\bigcup\left\{P_{\kappa}^{\mathrm{a}_{\alpha}}: \alpha \in \lambda\right\}$. Since $\left\{P_{\kappa}^{\mathrm{a}_{\alpha}}: \alpha \in \lambda\right\}$ is a strongly continuous <-increasing chain of ccc posets, it follows that $P$ is ccc. Furthermore $\wp(\mathbb{N}, P)$ is equal to the union of the increasing sequence $\left\{\wp\left(\mathbb{N}, P_{\kappa}^{a_{\alpha}}\right): \alpha<\lambda\right\}$. It then follows immediately from condition (6) that $\mathfrak{s}$ is forced to be $\lambda$. Similarly by Proposition 3.3. $P$ forces that $\mathfrak{b}=\lambda$. Now we check that $P$ forces that $M A(\kappa)$ holds: that is, if $Q$ is any $P$-name of a ccc poset of cardinality less than $\kappa$ and $\left\{A_{\xi}: \xi<\mu\right\}$ is a family of maximal antichains of $Q$ with $\mu<\kappa$, then there is a filter $G$ on $Q$ that meets each $A_{\xi}$. To show that this is forced by $P$, we may choose a $P$-name $\dot{Q}$ for $Q$ as well as $P$-name $\left\{\dot{A}_{\xi}: \xi<\kappa\right\}$ for the maximal antichains. We may assume that $1_{P}$ forces that $\dot{Q}$ is ccc. Since $\dot{Q}$ has cardinality less than $\lambda$, there is an $\alpha<\lambda$ such that $\dot{Q}$, and each $\dot{A}_{\xi}$ is a $P_{\kappa}^{\mathrm{a}_{\alpha}}$-name. Choose any $\gamma<\lambda$ so that $\dot{Q} * \operatorname{Fn}((\gamma, \gamma+\omega), 2)$ is not in the list $\left\{h(\zeta): \zeta \leq \zeta_{\alpha}\right\}$ and let $h\left(\zeta^{\prime}\right)=\dot{Q} * \operatorname{Fn}((\gamma, \gamma+\omega), 2)$. It follows from inductive conditions (1) and (7), that the sequence $\left\{\zeta_{\alpha}: \alpha \in \lambda\right\}$ is unbounded in $\lambda$. So we may choose limit $\delta<\lambda$ maximal so that $\zeta_{\delta} \leq \zeta^{\prime}$. Since $\zeta_{\delta+3}=\zeta_{\delta}$ and $\zeta_{\delta+\omega}$ is greater than $\zeta^{\prime}$, there is a minimal $n \geq 3$ such that $\zeta^{\prime}<\zeta_{\delta+n+1}$. It should be clear that by inductive condition (7) that $\zeta^{\prime}+1$ is equal to $\zeta_{\delta+n+1}$ and so $\dot{Q}_{\alpha_{\delta+n+1}}$ was chosen to be $\dot{Q} * \operatorname{Fn}((\gamma, \gamma+\omega), 2)$. This ensures that $P_{\kappa}^{\mathrm{a}_{\delta+n+1}}$ forces that there is a filter on $\dot{Q}$ that meets each $\dot{A}_{\xi}$.

Now let $\dot{\mathcal{U}}$ be a $P$-name of an ultrafilter on $\mathbb{N}$. As we did in the inductive step (3), we can let $\mathcal{E}$ be the set of all $\dot{e} \in \wp(\mathbb{N}, P)$ that are forced by $1_{P}$ to be an element of $\dot{\mathcal{U}}$. There is a cub $C \subset \lambda$ such that for all $\delta \in S_{\kappa}^{\lambda}, \mathcal{E} \cap \wp\left(\mathbb{N}, P_{\kappa}^{\mathrm{a}_{\delta}}\right)$ is a maximal set that is forced by $P_{\kappa}^{\mathrm{a} \delta}$ to be an ultrafilter on $\mathbb{N}$. Now let $X=\{\xi \in \lambda: h(\xi) \in \mathcal{E}\}$. We can pass to a cub subset $C^{\prime}$ of $C$ so that for all $\delta \in C^{\prime} \cap S_{\kappa}^{\lambda}$, the set $\{h(\xi): \xi \in X \cap \delta\}$ is equal to $\mathcal{E} \cap \wp\left(\mathbb{N}, P_{\kappa}^{\mathrm{a} \delta}\right)$. Now choose a $\delta \in E \cap C^{\prime}$ so that $X_{\delta}=X \cap \delta$. It follows from inductive step (3), that $\mathcal{E} \cap \mathcal{A}_{i}^{\text {a } \delta}$ is not empty for all $i<\kappa$. By Lemma 2.9, $P$ forces that $\dot{\mathcal{U}}$ has a subset of size $\kappa$ with no pseudo-intersection.

\section{ANOTHER CCC POSET FOR RAISING $\mathfrak{s}$}

The proper poset $\mathcal{Q}_{\text {Bould }}$ is introduced in [1] (also Sh:207 in the Shelah archive) to establish the consistency of $\mathfrak{b}<\mathfrak{s}=\mathfrak{a}$. For special directed subfamilies $\mathcal{D}$ of $\mathcal{Q}_{\text {Bould }}$, there is a ccc poset denoted $Q(\mathcal{D})$ that is analogous to $\mathbb{L}(\mathcal{E})$ for filters $\mathcal{E}$ on $\mathbb{N}$ (see Definition 5.6). Let us note the important properties of $\mathcal{Q}_{\text {Bould }}$ shown to hold in [11. The first is that it adds an unsplit real.

Proposition 5.1. If $\dot{L}$ is the generic subset of $\mathbb{N}$ added by $\mathcal{Q}_{\text {Bould, }}$ then the set $\left\{A \subset \mathbb{N}: A \in V\right.$ and $\left.|\dot{L} \backslash A|<\aleph_{0}\right\}$ is a free ultrafilter over $V \cap \mathcal{P}(\mathbb{N})$.

The second is that the forcing does not add a dominating real. By Lemma 2.8 , this property is needed if such a $Q(\mathcal{D})$ is to replace $\mathbb{L}(\mathcal{E})$ in constructing a in APv.

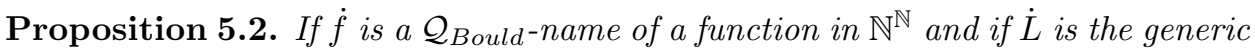
subset of $\mathbb{N}$ added by $\mathcal{Q}_{\text {Bould, }}$, then there is a (ground model) $h \in \mathbb{N}^{\mathbb{N}}$ so that, for every infinite set $A \subset \mathbb{N}$ in the ground model, the set $\{n \in A: \dot{f}(n)<h(n)\}$ will be forced to be infinite. 
We adopt the elegant representation of this poset from [1. Also many of the technical details for constructing ccc subposets of this poset, sharing the above mentioned properties, are similar to the results in [9]. The main tool is to utilize logarithmic measures.

Definition 5.3. A function $h$ is a logarithmic measure on a set $S \subset \mathbb{N}$ if $h$ is a function from $[S]^{<\aleph_{0}}$ into $\omega$ with the property that whenever $\ell \geq 0$ and $h(a \cup b) \geq$ $\ell+1$, then either $h(a) \geq \ell$ or $h(b) \geq \ell$. A pair $(s, h) \in \mathcal{L}_{n}$ if $s \in[\mathbb{N}]^{<\aleph_{0}}$ and $h$ is a logarithmic measure on $s$ with $h(s) \geq n$. The elements e of $[\mathbb{N}]^{<\aleph_{0}}$ such that $h(e)>0$ are called the positive sets.

When we discuss $t \in \mathcal{L}_{1}$, we use $\operatorname{int}(t)$ and $h_{t}$ to denote the pair where $t=$ $\left(\operatorname{int}(t), h_{t}\right)$. We say that a subset $e$ of $\operatorname{int}(t)$ is $t$-positive to mean that $h_{t}(e)>0$. Note that if $(s, h) \in \mathcal{L}_{n}$ and $\emptyset \neq e \subset s$, then $\left(e, h \uparrow[e]^{<\aleph_{0}}\right) \in \mathcal{L}_{h(e)}$.

Definition 5.4. The poset $\mathcal{Q}_{\text {Bould }}$ consists of all pairs $(u, T)$ where

(1) $u \in[\mathbb{N}]<\aleph_{0}$,

(2) $T=\left\{t_{\ell}: \ell \in \omega\right\}$ is a sequence of members of $\mathcal{L}_{1}$ where for each $\ell$, $\max \left(\operatorname{int}\left(t_{\ell}\right)\right)<\min \left(\operatorname{int}\left(t_{\ell+1}\right)\right)$, and the sequence $\left\{h_{t_{\ell}}\left(\operatorname{int}\left(t_{\ell}\right)\right): \ell \in \omega\right\}$ is monotone increasing and unbounded.

For each $(u, T) \in \mathcal{Q}_{\text {Bould }}$, let $\ell_{u, T}$ be the minimal $\ell$ such that $\max (u)<\min \left(\operatorname{int}\left(t_{\ell}\right)\right)$ and let $\operatorname{int}(u, T)=\bigcup\left\{\operatorname{int}\left(t_{\ell}\right): \ell_{u, T} \leq \ell\right\}$.

For $T_{1}=\left\{t_{\ell}^{1}: \ell \in \omega\right\}$ and $T_{2}=\left\{t_{\ell}^{2}: \ell \in \omega\right\}$ with $\left(u_{1}, T_{1}\right),\left(u_{2}, T_{2}\right) \in \mathcal{Q}_{\text {Bould }}$, the extension relation is defined by $\left(u_{2}, T_{2}\right) \geq\left(u_{1}, T_{1}\right)$ (stronger) providing

(1) $u_{2} \supset u_{1}$ and $u_{2} \backslash u_{1}$ is contained in $\operatorname{int}\left(u_{1}, T_{1}\right)$,

(2) $\operatorname{int}\left(u_{2}, T_{2}\right) \subset \operatorname{int}\left(u_{1}, T_{1}\right)$

(3) there is a sequence of finite subsets of $\omega,\left\langle B_{k}: k \in \omega\right\rangle$, such that for each $k \geq \ell_{u_{2}, T_{2}}, \max \left(B_{k}\right)<\min \left(B_{k+1}\right)$ and int $\left(t_{k}^{2}\right) \subset \bigcup\left\{\operatorname{int}\left(t_{\ell}^{1}\right): \ell \in B_{k}\right\}$,

(4) for every $k \geq \ell_{u_{2}, T_{2}}$ and every $t_{k}^{2}$-positive $e \subset \operatorname{int}\left(t_{k}^{2}\right)$ there is a $j \in B_{k}$ such that $e \cap \operatorname{int}\left(t_{j}^{1}\right)$ is $t_{j}^{1}$-positive.

For a finite subset $\mathcal{D}$ of $\mathcal{Q}_{\text {Bould }}$ and an element $t$ of $\mathcal{L}_{1}$, we say that $t$ is built from $\mathcal{D}$ if there is a $q=\left(\emptyset, T_{q}\right) \in \mathcal{Q}_{\text {Bould }}$ with $t \in T_{q}$ such that $q \geq\left(\emptyset, T_{q^{\prime}}\right)$ for each $q^{\prime}=\left(u_{q^{\prime}}, T_{q^{\prime}}\right) \in \mathcal{D}$.

Definition 5.5. The elements $q \in \mathcal{Q}_{\text {Bould }}$ of the form $\left(\emptyset, T_{q}\right)$ are called pure conditions. We let $\mathcal{P}_{\text {Bould }}$ denote the set of all pure conditions in $\mathcal{Q}_{\text {Bould. }}$ A family $\mathcal{D} \subset \mathcal{P}_{\text {Bould }}$ is finitely compatible if each finite subset of $\mathcal{D}$ has an upper bound in $\mathcal{Q}_{\text {Bould. }}$ The family $\mathcal{D}$ is finitely directed if each finite subset has an upper bound in $\mathcal{D}$.

For an element $q \in \mathcal{Q}_{\text {Bould }}$, we let $u_{q}$ and $T_{q}$ denote the elements with $q=$ $\left(u_{q}, T_{q}\right)$. We also use $\operatorname{int}(q)$ for $\operatorname{int}\left(u_{q}, T_{q}\right)$. The fact that the elements of $T_{q}$ are enumerated by $\omega$ is unimportant. It will be convenient to adopt the convention that for an infinite set $L \subset \omega$, the sequence $\left(\emptyset,\left\{t_{\ell}: \ell \in L\right\}\right)$ is a pure condition so long as $\left(\emptyset,\left\{t_{n}^{\prime}: n \in \omega\right\}\right) \in \mathcal{Q}_{\text {Bould }}$ where $\left\{\ell_{n}: n \in \omega\right\}$ is the increasing enumeration of $L$ and $t_{n}^{\prime}=t_{\ell_{n}}$ for each $n \in \omega$.

Definition 5.6. If $\mathcal{D}$ is a finitely directed set of pure conditions, then define $Q(\mathcal{D})$ to be the subposet $\left\{(u, T): u \in[\mathbb{N}]^{<\aleph_{0}},(\emptyset, T) \in \mathcal{D}\right\}$ of $\mathcal{Q}_{\text {Bould. }}$. We let $\dot{L}_{\mathcal{D}}$ denote the $Q(\mathcal{D})$-name $\left\{(n, q): q \in Q(\mathcal{D}), n \in u_{q}\right\}$. 
Proposition 5.7. If $\mathcal{D} \subset \mathcal{P}_{\text {Bould }}$ is finitely directed, then $Q(\mathcal{D})$ is a $\sigma$-centered poset. Each $q \in Q(\mathcal{D})$ forces that $\dot{L}_{\mathcal{D}} \backslash \operatorname{int}\left(T_{q}\right) \subset u_{q}$.

It follows from the results in 9 that there is a $\operatorname{Fn}\left(2^{\omega}, 2\right)$-name $\dot{\mathcal{D}}$ that is forced to be a finitely directed subset of $\mathcal{P}_{\text {Bould }}$ with the property that $\operatorname{Fn}\left(2^{\omega}, 2\right) * Q(\dot{\mathcal{D}})$ will add an unsplit real and not add a dominating real (see Lemma 5.16 and Lemma 5.22). These will be the factor posets we will use in place of $\mathbb{L}(\mathcal{D})$ in the construction of members of APv. We will also need an analogue of Lemma 3.6 and we now introduce a condition on $\mathcal{D}$ that will ensure that $Q(\mathcal{D})<_{V} Q\left(\mathcal{D}_{2}\right)$ when $\mathcal{D}_{2} \supset \mathcal{D}$ in a forcing extension of $V$ (see Proposition 5.11).

Definition 5.8. Let $\mathcal{D} \subset \mathcal{P}_{\text {Bould }}$ be directed mod finite. For a set $E=\left\{\left(u_{n}, T_{n}\right)\right.$ : $n \in \omega\} \subset Q(\mathcal{D})$ we say that $\left(\emptyset,\left\{t_{\ell}: \ell \in \omega\right\}\right) \in \mathcal{P}_{\text {Bould }}$, is a mod finite meet of $E$ if, for each $0<\ell \in \omega, w \subset \max \left(\right.$ int $\left.\left(t_{\ell-1}\right)\right)$, and $h_{\ell}$-positive $e \subset$ int $\left(t_{\ell}\right)$, there is an $n<\min i n t\left(t_{\ell+1}\right)$ and $a w_{e} \subset e$ such that $\left(w \cup w_{e},\left\{t_{m}: m>\ell\right\}\right) \geq\left(u_{n}, T_{k}\right)$ for each $k \leq \max \left\{n, \ell, \max \left(\operatorname{int}\left(t_{\ell-1}\right)\right)\right\}$.

A set of pure conditions $\mathcal{D}$ is $\aleph_{1}$-directed mod finite if it is directed mod finite and each predense subset of $Q(\mathcal{D})$ has a mod finite meet in $\mathcal{D}$.

Lemma 5.9. Suppose that $\left\{\left(u_{n}, T_{n}\right): n \in \omega\right\}$ is a subset of $\mathcal{Q}_{\text {Bould }}$ and let $T$ be a mod finite meet. Then, for each $w \in[\mathbb{N}]^{<\aleph_{0}},\left\{\left(u_{n}, T_{n}\right): n \in \omega\right\}$ is predense below $(w, T)$ in all of $\mathcal{Q}_{\text {Bould }}$.

Proof. Let $\left(w, T^{\prime}\right)$ be an arbitrary member of $\mathcal{Q}_{\text {Bould }}$ that is compatible with $(w, T)=\left(w,\left\{t_{\ell}: \ell \in \omega\right\}\right)$ in $\mathcal{Q}_{\text {Bould }}$. By extending $\left(w, T^{\prime}\right)$, we may assume that $\left(w, T^{\prime}\right)<\left(w,\left\{t_{\ell}: \ell>\ell_{w}\right\}\right)$, where $w \subset \min \left(\operatorname{int}\left(t_{\ell_{w}}\right)\right)$. Choose any $T^{\prime}$ positive $e$ so that $\left(w \cup e, T^{\prime}\right)<\left(w, T^{\prime}\right)$ and $\max (w)<\min (e)$. Therefore there is an $\ell>\ell_{w}$ such that $h_{\ell}\left(e \cap \operatorname{int}\left(t_{\ell}\right)\right)>0$, and so, by Definition 5.8, there is an $n<\min \left(\operatorname{int}\left(t_{\ell+1}\right)\right)$ and a $w_{e} \subset e$ so that $\left(w \cup w_{e}, T\right)<\left(u_{n}, T_{n}\right)$ and we have that $\left(w \cup w_{e}, T^{\prime}\right)<(w, T)<\left(u_{n}, T_{n}\right)$.

Definition 5.10. $\mathbb{Q}_{207}$ is the set of $Q(\mathcal{D})$ where $\mathcal{D}$ is an $\aleph_{1}$-directed mod finite set of pure conditions.

Proposition 5.11. Assume $\left\{P_{i}: i<\kappa\right\}$ is a continuous $<-$-chain of ccc posets and that $\left\{\dot{Q}_{i}: i<\kappa\right\}$ is a chain such that, for each $i<\kappa, \dot{Q}_{i}$ is a $P_{i}$-name of a member of $\mathbb{Q}_{207}$, then $\left\{P_{i} * \dot{Q}_{i}: i<\kappa\right\}$ is a continuous $<\cdot$-chain of ccc posets.

Proof. By Lemma 1.5, it suffices to prove that each $P_{j}$-name of a predense subset of $\dot{Q}_{j}$ is forced by $P_{i}$ to be predense in $\dot{Q}_{i}$. Since $\dot{Q}_{j}$ is forced to be a subset of $\dot{Q}_{i}$, it is immediate that $[\mathbb{N}]^{<\aleph_{0}} \times\{T\}$ is a predense subset of $\dot{Q}_{i}$ for each $(\emptyset, T) \in \dot{Q}_{j}$. Now the Proposition follows by Lemma 5.9 .

Definition 5.12. Say that a subset $\tilde{\mathcal{L}}$ of $\mathcal{L}_{1}$ is $\mathcal{D}$-positive if for each finite $\mathcal{D}^{\prime} \subset \mathcal{D}$ and each $n \in \omega$, there is a $t \in \tilde{\mathcal{L}} \cap \mathcal{L}_{n}$ that is built from $\mathcal{D}^{\prime}$.

Proposition 5.13. If $\tilde{\mathcal{L}} \subset \mathcal{L}_{1}$ is $\mathcal{D}$-positive for some $\mathcal{D} \subset \mathcal{P}_{\text {Bould }}$, then for each finite $\mathcal{D}^{\prime} \subset \mathcal{D}$, the set $\left\{t \in \tilde{\mathcal{L}}: t\right.$ is built from $\left.\mathcal{D}^{\prime}\right\}$ is $\mathcal{D}$-positive.

The poset $\operatorname{Fn}(\mathbb{N}, 2)$ is forcing isomorphic to the poset $\omega^{<\omega}$ ordered by extension. Similarly, each infinite branching (non-empty) subset $S \subset \omega^{<\omega}$ is forcing isomorphic to $\omega^{<\omega}$; we say that $S$ is infinite branching if, for each $s \in S$, the $\{n \in \omega: s \frown n \in S\}$ is infinite. For such infinite branching $S \subset \omega^{<\omega}$ and each $k \in \omega$, let $\dot{n}_{k}^{S}$ denote the $S$-name $\{(s(k), s): s \in S$ and $k \in \operatorname{dom}(s)\}$. 
Definition 5.14. Fix an enumerating function $\lambda$ from $\omega$ onto $\mathcal{L}_{1}$. For $\mathcal{D} \subset \mathcal{P}_{\text {Bould }}$, say that $S \subset \omega^{<\omega}$ is $\mathcal{D}^{+}$-branching if $\emptyset \in S$ and, for each $s \in S$,

(1) for each $k \in \operatorname{dom}(s), \lambda(s(k)) \in \mathcal{L}_{k}$,

(2) $\max (\operatorname{int}(\lambda(s(j))))<\min (\operatorname{int}(\lambda(s(k))))$ for $j<k \in \operatorname{dom}(s)$, and

(3) the set $\{\lambda(n): s \frown n \in S\}$ is a $\mathcal{D}$-positive set.

For each $k \in \omega$, define the $S$-name $\dot{r}_{k}^{S}$ to be $\lambda\left(\dot{n}_{k}^{S}\right)$. For each finite $\mathcal{D}^{\prime} \subset \mathcal{P}_{\text {Bould }}$, let $\dot{I}_{\mathcal{D}^{\prime}}^{S}$ be the $S$-name for the set $\left\{k \in \omega: \dot{r}_{k}^{S}\right.$ is built from $\left.\mathcal{D}^{\prime}\right\}$.

Lemma 5.15. If $\mathcal{D} \subset \mathcal{P}_{\text {Bould }}$ is finitely compatible and if $S \subset \omega^{<\omega}$ is $\mathcal{D}^{+}$-branching then $\Vdash_{S} \dot{\mathcal{D}}^{S}=\mathcal{D} \cup\left\{\left(\emptyset,\left\{\dot{r}_{k}^{S}: k \in \dot{I}_{\mathcal{D}^{\prime}}^{S}\right\}\right): \mathcal{D}^{\prime} \in[\mathcal{D}]^{<\aleph_{0}}\right\}$ is finitely directed.

Lemma 5.16. If $\mathcal{D} \subset \mathcal{P}_{\text {Bould }}$ is finitely directed, then there is a $\operatorname{Fn}(\mathbb{N}, 2)$-name $\dot{\mathcal{D}}_{1}$ such that

(1) $\Vdash_{\mathrm{Fn}(\omega, 2)} \mathcal{D} \subset \dot{\mathcal{D}}_{1} \subset \mathcal{P}_{\text {Bould }}$ and $\dot{\mathcal{D}}_{1}$ is finitely directed,

(2) for each $A \subset \mathbb{N}, \Vdash_{\mathrm{Fn}(\mathbb{N}, 2)}\left(\exists q \in \dot{\mathcal{D}}_{1}\right)(\operatorname{int}(q) \subset A$ or $\operatorname{int}(q) \cap A=\emptyset)$

Proof. Let $S \subset \omega^{<\omega}$ be the maximal $\mathcal{D}^{+}$-branching set. That is, $S$ is the set of all $s \in \omega^{<\omega}$ that satisfy properties (1) and (2) of Definition 5.14. For finite subsets $\mathcal{D}^{\prime}$ of $\mathcal{D}$, let $\dot{I}_{\mathcal{D}^{\prime}}^{S}$ be an $S$-name for the set $\left\{k \in \omega: \dot{r}_{k}^{S}\right.$ is built from $\left.\mathcal{D}^{\prime}\right\}$.

For a subset $A$ of $\mathbb{N}$, define $\mathcal{L}(A)$ to be $\left\{t \in \mathcal{L}_{1}: \operatorname{int}(t) \subset A\right\}$. If $\mathcal{L}(A)$ is not $\mathcal{D}$-positive, there is a finite $\mathcal{D}_{A} \subset \mathcal{D}$ and an integer $m$ such that there is no $t \in \mathcal{L}_{m} \cap \mathcal{L}(A)$ that is built from $\mathcal{D}_{A}$. Since, for each $t \in \mathcal{L}_{n+1}$, there is an $e \subset \operatorname{int}(t)$ such that $h_{t}(e) \geq n$ and either $e \subset A$ or $e \subset(\mathbb{N} \backslash A)$, it follows that if $\mathcal{A}$ is a finite partition of $\mathbb{N}$, then $\mathcal{L}(A)$ is $\mathcal{D}$-positive for some $A \in \mathcal{A}$. Therefore, by Zorn's Lemma, there is a free ultrafilter $\mathcal{U}$ on $\mathbb{N}$ so that $\mathcal{L}(U)$ is $\mathcal{D}$-positive for all $U \in \mathcal{U}$. For each $U \in \mathcal{U}$, let $\dot{I}_{U}^{S}$ denote an $S$-name that will evaluate to $\left\{k \in \omega: \operatorname{int}\left(\dot{r}_{k}^{S}\right) \subset U\right\}$. It follows from the fact that $\mathcal{L}(U)$ is $\mathcal{D}$ positive, that $\Vdash_{S} \dot{I}_{U}^{S} \cap \dot{I}_{\mathcal{D}^{\prime}}^{S}$ is infinite for each $\mathcal{D}^{\prime} \in[\mathcal{D}]^{<\aleph_{0}}$. It is also clear that $\Vdash_{S}\left\{\dot{I}_{U}^{S} \cap \dot{I}_{\mathcal{D}^{\prime}}^{S}: U \in \mathcal{U}\right\}$ is closed under finite intersections. It then follows that $\Vdash_{S} \dot{\mathcal{D}}^{S}=\mathcal{D} \cup\left\{\left(\emptyset,\left\{\dot{r}_{k}^{S}: k \in \dot{I}_{\mathcal{D}^{\prime}}^{S}\right\}\right): \mathcal{D}^{\prime} \in[\mathcal{D}]^{<\aleph_{0}}\right\}$ is the desired finitely directed subset of $\mathcal{P}_{\text {Bould }}$.

In order to produce extensions of finitely directed $\mathcal{D} \subset \mathcal{P}_{\text {Bould }}$ that are $\aleph_{1}$ directed mod finite, we will need the following tools for constructing members of $\mathcal{L}_{n}$ for arbitrarily large $n$. A family $L \subset[\mathbb{N}]^{<\aleph_{0}}$ naturally induces a logarithmic measure.

Definition 5.17. Let $L \subset[\mathbb{N}]^{<\aleph_{0}}$ and define the relation $h(s) \geq \ell$ for $s \in[\mathbb{N}]^{<\aleph_{0}}$ by induction on $|s|$ and $\ell$ as follows:

(1) $h(e) \geq 0$ for all $e \in[\mathbb{N}]^{<\aleph_{0}}$,

(2) $h(e)>0$ if e contains some non-empty element of $L$,

(3) for $\ell>0, h(e) \geq \ell+1$ if and only if, $|e|>1$ and whenever $e_{1}, e_{2} \subset e$ are such that $e=e_{1} \cup e_{2}$ then $h\left(e_{1}\right) \geq \ell$ or $h\left(e_{2}\right) \geq \ell$.

The definition of $h(e)$ is the maximum $\ell$ such that $h(e) \geq \ell$.

Proposition 5.18 ([1, Lemma 4.7]). Let $L \subset[\mathbb{N}]^{<\aleph_{0}}$ be an upward closed family of non-empty sets and let $h$ be the associated logarithmic measure. Assume that whenever $\mathbb{N}$ is partitioned into finitely many sets $\mathcal{A}$, there is some $A \in \mathcal{A}$ such that $L \cap[A]^{<\aleph_{0}}$ is non-empty. Then, for any partition $\mathcal{A}$ of $\mathbb{N}$, and any integer $n$, there is an $A \in \mathcal{A}$ and an $e \subset A$ such that $h(e) \geq n$. 
Lemma 5.19. If $\mathcal{D} \subset \mathcal{P}_{\text {Bould }}$ is finitely directed and $E=\left\{\left(u_{n}, T_{n}\right): n \in \omega\right\}$ is a subset of $Q(\mathcal{D})$, then in the forcing extension by $\mathrm{Fn}(\mathbb{N}, 2)$, there is a finitely directed $\mathcal{D} \subset \mathcal{D}_{1} \subset \mathcal{P}_{\text {Bould }}$ such that either $E$ is not predense in $Q\left(\mathcal{D}_{1}\right)$ or there is a condition $(\emptyset, T) \in \mathcal{D}_{1}$ such that $(\emptyset, T)$ is the mod finite meet of $E$.

Proof. We assume that in the forcing extension by $\mathrm{Fn}(\mathbb{N}, 2), E$ is a predense subset of $Q\left(\mathcal{D}_{1}\right)$ for each finitely directed $\mathcal{D}_{1}$ with $\mathcal{D} \subset \mathcal{D}_{1} \subset \mathcal{P}_{\text {Bould }}$. We will prove there is a $\mathcal{D}^{+}$-branching $S \subset \omega^{<\omega}$ satisfying that $\Vdash_{S}\left(\emptyset,\left\{\dot{r}_{k}^{S}: k \in \omega\right\}\right)$ is a mod finite meet of $E$. By Lemma 5.15, $S$ forces that there is a $\mathcal{D}_{1}$ as required. Since $S$ will be forcing isomorphic to $\operatorname{Fn}(\mathbb{N}, 2)$, this will complete the proof.

Let $L=\left\{w \in[\mathbb{N}]^{<\aleph_{0}}:(\exists n \in \omega) u_{n} \subset w\right\}$, and for each $w \in[\mathbb{N}]^{<\aleph_{0}}$, let

$$
L_{w}=\left\{w_{1} \in[\mathbb{N}]^{<\aleph_{0}}: \max (w)<\min \left(w_{1}\right) \text { and } w \cup w_{1} \in L\right\} .
$$

Say that $t \in \mathcal{L}_{1}$ is $(E, \ell)$-large (for $\ell \in \mathbb{N}$ ) if $\ell<\min (\operatorname{int}(t))$ and for each $w \subset$ $\{1, \ldots, \ell\}$ each $t$-positive set contains an element of $L_{w}$. For each $(E, \ell)$-large $t$, let $N_{t} \geq \max (\operatorname{int}(t))$ denote a sufficiently large integer such that each $t$-positive set contains an element of $\left\{u_{n}: n<N_{t}\right\}$.

Claim 1. For each $\ell \in \mathbb{N}$, the set $\left\{t \in \mathcal{L}_{\ell}: t\right.$ is $(E, \ell)$-large $\}$ is $\mathcal{D}$-positive.

Proof of Claim 1 . Let $\ell \in \mathbb{N}$. Since $\mathcal{D}$ is finitely directed, in order to show that the set of $(E, \ell)$-large elements is $\mathcal{D}$-positive, it suffices to show that for each $q \in \mathcal{D}$ there is a $(E, \ell)$-large $t$ that is built from $q$. Note $t \in \mathcal{L}_{1}$ is built from $q$ if $\operatorname{int}(t) \subset \operatorname{int}(q)$ and, for each $t$-positive set $e$, there is a $t_{e} \in T_{q}$ such that $e \cap i n t\left(t_{e}\right)$ is $t_{e}$-positive. Say that a finite set $e$ is $q$-positive if there is a $t_{e} \in T_{q}$ such that $e \cap \operatorname{int}\left(t_{e}\right)$ is $t_{e}$-positive. Note also that if $q_{1}, q \in \mathcal{P}_{\text {Bould }}$ and $q_{1} \geq q$, then each $q_{1}$-positive set is also $q$-positive.

Define $L_{q, \ell}$ to be the elements of $\bigcap_{w \subset\{1, \ldots, \ell\}} L_{w}$ that are also $q$-positive. Let $h_{q, \ell}$ denote the associated logarithmic measure as in Definition 5.17. If $e \in L_{q, \ell}$ is a subset of $\operatorname{int}(q)$, then $\left(e, h_{q, \ell}\left\lceil[e]^{<\aleph_{0}}\right)\right.$ is built from $q$. Therefore, to finish the proof of the claim it will suffice to prove that there is an $e \in L_{q, \ell}$ with $h_{q, \ell}(e)>\ell$. We prove this using Proposition 5.18 so let $\mathcal{A}$ be a finite partition of $\mathbb{N}$. Pass to the forcing extension by $\operatorname{Fn}(\mathbb{N}, 2)$ and choose, by Corollary [5.16, a finitely directed $\mathcal{D}_{1} \subset$ $\mathcal{P}_{\text {Bould }}$ that contains $\mathcal{D}$ and satisfies that there is a $q_{1} \geq q$ in $Q\left(\mathcal{D}_{1}\right)$ and an $A \in \mathcal{A}$ such that $\operatorname{int}\left(q_{1}\right) \subset A$. We may arrange that $\ell<\min \left(\operatorname{int}\left(q_{1}\right)\right)$. By assumption, $E$ is a predense subset of $Q\left(\mathcal{D}_{1}\right)$. Let $\left\{t_{k}: k \in \omega\right\}$ be the standard enumeration of $T_{q_{1}}$. For each $w \subset\{1, \ldots, \ell\}$, there is a $q_{w} \geq\left(w, T_{q_{1}}\right)$ and an $n_{w} \in \omega$ such that $q_{w} \geq\left(u_{n_{w}}, T_{n_{w}}\right)$. There is a $K_{w} \in \omega$ such that $\left(u_{q_{w}} \backslash w\right) \subset \bigcup\left\{\operatorname{int}\left(t_{k}\right): k \in K_{w}\right\}$. Since $u_{q_{w}} \supset u_{n_{w}}$, we have that any finite set containing $u_{q_{w}}$ is in $L_{w}$. This shows that, for some $K \in \omega, e=\bigcup_{k<K} i n t\left(t_{k}\right)$ is in $L_{w}$ for each $w \subset\{1, \ldots, \ell\}$. Since $e$ is $q_{1}$-positive and $q_{1} \geq q$, it follows that $e$ is $q$-positive. This completes the proof that $e \in L_{q, \ell}$.

For each $s \in \omega^{<\omega}$, define $\ell_{s}$ to be the maximum element of the set $\{1\} \cup$ $\bigcup_{k \in \operatorname{dom}(s)} \operatorname{int}(\lambda(s(k)))$. Now define the infinite branching $S \subset \omega^{<\omega}$ by the recursive rule that, for each $s \in S$ and $n \in \omega, s \frown n \in S$ if and only if

(1) $\lambda(n)$ is $\left(E, \ell_{s}\right)$-large, and

(2) $\lambda(n)$ is built from $\left\{\left(\emptyset, T_{n}\right): n<\max \left\{N_{s(k)}: k \in \operatorname{dom}(s)\right\}\right\}$ and $\left(\emptyset, T_{\ell_{s}}\right)$.

Claim 1 and Proposition 5.13 show that $S$ is $\mathcal{D}^{+}$-branching. The definition of the notion of being $(E, \ell)$-large and the second criterion of being an element of $S$, 
ensures that $\Vdash_{S}\left(\emptyset,\left\{\dot{r}_{k}^{S}: k \in \omega\right\}\right)$ is the $\bmod$ finite meet of $E$. Since $\operatorname{Fn}(\mathbb{N}, 2)$ is forcing isomorphic to $S$, the proof of the Lemma now follows from Lemma 5.15.

The next result follows by first applying Lemma 5.15 to obtain directed mod finite extension of $\mathcal{D}$, next applying Lemma 5.16, and finally repeatedly applying Lemma 5.19 in a recursive construction of length $\mathfrak{c}$.

Lemma 5.20. If $\mathcal{D}$ is a finitely compatible set of pure conditions then there is a $\operatorname{Fn}(\mathfrak{c} \times \mathbb{N}, 2)$-name $\dot{\mathcal{D}}_{1}$ such that $\operatorname{Fn}(\mathfrak{c} \times \mathbb{N}, 2)$ forces that

(1) $\dot{\mathcal{D}}_{1} \subset \mathcal{P}_{\text {Bould }}$ is finitely directed and includes $\mathcal{D}$,

(2) $Q\left(\dot{\mathcal{D}}_{1}\right)$ is in $\mathbb{Q}_{207}$, and

(3) the ground model subsets of $\mathbb{N}$ is not a splitting family in the further forcing extension by $Q\left(\dot{\mathcal{D}}_{1}\right)$.

We now establish notation that will be useful when preserving that a family of names is forced to be very thin.

Definition 5.21. Let $Q \in \mathbb{Q}_{207}$ and let $\dot{f}$ be a $Q$-name such that $\Vdash_{Q} \dot{f} \in \mathbb{N}^{\mathbb{N}}$. A condition $q \in Q$ is $\dot{f}$-ready if, for each integer $\ell>0, w \subset\left\{1, \ldots, \max \left(\operatorname{int}\left(t_{\ell-1}^{q}\right)\right)\right\}$, and $t_{\ell}^{q}$-positive $e$, there is a $w_{e} \subset$ e such that $\left(w \cup w_{e},\left\{t_{k}^{q}: k>\ell\right\}\right)$ decides the value of $\dot{f}(j)<\min \left(\operatorname{int}\left(t_{\ell+1}^{q}\right)\right)$ for each $j \in\left\{1, \ldots, \max \left(\operatorname{int}\left(t_{\ell-1}^{q}\right)\right)\right\}$.

Lemma 5.22. For each $Q \in \mathbb{Q}_{207}$ and $Q$-name $\dot{f}$ such that $\Vdash_{Q} \dot{f} \in \mathbb{N}^{\mathbb{N}}$, the set of $\dot{f}$-ready conditions is a dense subset of $Q$.

Proof. Let $Q$ and $\dot{f}$ be as in the statement of the Lemma and let $q$ be any element of $Q$. For each $k \in \mathbb{N}$, there is a pre-dense set $\left\{\left(u_{n}^{k}, T_{n}^{k}\right): n \in \omega\right\} \subset Q$ satisfying that $\left(u_{q}, T_{n}^{k}\right) \geq q$ and $\left(u_{n}^{k}, T_{n}^{k}\right)$ forces a value on $\dot{f}\lceil\{1, \ldots, k\}$ for each $n \in \omega$. By choosing a cofinite subset of $T_{n}^{k}$ we may assume also that $\left(u_{n}^{k}, T_{n}^{k}\right)$ forces that the range of $\dot{f} \uparrow\{1, \ldots, k\}$ is contained in $\min \left(\operatorname{int}\left(T_{n}^{k}\right)\right)$. Since $Q \in \mathbb{Q}_{207}$, there is, for each $k \in \mathbb{N}$, a condition $\left(\emptyset,\left\{t_{\ell}^{k}: \ell \in \omega\right\}\right) \in Q$ which is the mod finite meet of the predense set $\left\{\left(u_{n}^{k}, T_{n}^{k}\right): n \in \omega\right\}$. We recall that this means that for each $\ell \in \omega, w \subset\left\{1, \ldots \max \left(\operatorname{int}\left(t_{\ell-1}^{k}\right)\right)\right\}$ and $t_{\ell}^{k}$-positive $e$, there is a $w_{e} \subset e$, such that $\left(w \cup w_{e},\left\{t_{m}^{k}: \ell<m \in \omega\right\}\right)$ decides the value of $\dot{f} \uparrow\{1, \ldots, k\}$. Also, if $n$ was the value witnessing that $\left(w \cup w_{e},\left\{t_{m}^{k}: \ell<m \in \omega\right\}\right) \geq\left(u_{n}^{k}, T_{n}^{k}\right)$ then the range of $\dot{f} \uparrow\{1, \ldots, k\}$ is also forced to be contained in $\min \left(\operatorname{int}\left(T_{n}^{k}\right)\right) \leq \min \left(\operatorname{int}\left(t_{\ell+1}^{k}\right)\right)$.

Let $T_{0}=\left\{t_{\ell}^{q}: \ell \in \omega\right\}$ and for each $k \in \mathbb{N}$, let $T_{k}=\left\{t_{\ell}^{k}: \ell \in \omega\right\}$. Choose $\left(\emptyset,\left\{t_{\ell}: \ell \in \omega\right\}\right)$ to be a mod finite meet of the family $\left\{\left(\emptyset, T_{k}\right): k \in \omega\right\}$. It follows easily from the definition that $\left(\emptyset,\left\{t_{\ell}: \max \left(u_{q}\right)<\ell \in \omega\right\}\right)$ is also a mod finite meet of this family, and so by re-indexing, we can assume that $t_{\ell}$ is built from a finite subset of $T_{\max \left(i n t\left(t_{\ell-1}\right)\right)}$ for each $\ell \in \omega$, and that $\max \left(u_{q}\right)<\min \left(\operatorname{int}\left(t_{0}\right)\right)$. We check that $\left(u_{q},\left\{t_{\ell}: \ell \in \omega\right\}\right)$ is $\dot{f}$-ready. Consider any $\ell>0$ and let $\bar{\ell}$ denote $\max i n t\left(t_{\ell-1}\right)$. Choose any $w \subset\{1, \ldots, \bar{\ell}\}$ and $t_{\ell^{-}}$positive $e$. Choose any $k$ so that $e \cap \operatorname{int}\left(t_{k}^{\bar{\ell}}\right)$ is $t_{k^{-}}^{\overline{-}}$ positive. Choose $w_{e} \subset\left(e \cap \operatorname{int}\left(t_{k}^{\bar{\ell}}\right)\right)$ so that $\left(w \cup w_{e},\left\{t_{m}^{\bar{\ell}}: k<m \in \omega\right\}\right)$ decides the value of $\dot{f}\left\lceil\{1, \ldots, \bar{\ell}\}\right.$. Since $\left(\emptyset,\left\{t_{m}: m \in \omega\right\}\right)$ is a mod finite meet of the sequence $\left\{\left(\emptyset, T_{m}\right): m \in \omega\right\}$, we also have that $\left(\emptyset,\left\{t_{m}: \ell<m \in \omega\right\}\right) \geq\left(\emptyset,\left\{t_{m}^{\bar{\ell}}: k<m \in \omega\right\}\right)$. Therefore $\left(w \cup w_{e},\left\{t_{m}: \ell<m \in \omega\right\}\right) \geq\left(w \cup w_{e},\left\{t_{m}^{\bar{\ell}}: k<m \in \omega\right\}\right)$. This proves that $\left(w \cup w_{e},\left\{t_{m}: \ell<m \in \omega\right\}\right)$ decides the value of $\dot{f}\lceil\{1, \ldots, \ell\}$. 
Corollary 5.23. Suppose that $\mathrm{a} \in \mathrm{APv}$ and let $\left|P_{\kappa}^{\mathrm{a}}\right| \leq \theta=\theta^{\aleph_{0}}<\lambda$ and let $\mathrm{a}_{1}$ denote the Cohen ${ }^{\theta}$-extension of $\mathrm{a}$. Then there is $a \mathrm{~b} \in \mathrm{APv}$ and a sequence $\left\{\mathcal{D}_{i}: i<\kappa\right\}$ satisfy that, for $i<j<\kappa$,

(1) $\mathrm{a}_{1} \leq_{\mathrm{AP}}^{0} \mathrm{~b}$ and $\mathcal{A}_{i}^{\mathrm{b}}=\mathcal{A}_{i}^{\mathrm{a}}$,

(2) $\mathcal{D}_{i} \subset \wp\left(\mathcal{L}_{1}, P_{i}^{\mathrm{a}_{1}}\right)$,

(3) $\mathcal{D}_{i} \subset \mathcal{D}_{j}$,

(4) $P_{i}^{\mathrm{a}_{1}}$ forces that $\{\emptyset\} \times \mathcal{D}_{i}=\left\{(\emptyset, T): T \in \mathcal{D}_{i}\right\}$ is a subset of $\mathcal{P}_{\text {Bould }}$ and is $\aleph_{1}$-directed mod finite,

(5) $P_{i}^{\mathrm{b}}=P_{i}^{\mathrm{a}_{1}} * Q\left(\{\emptyset\} \times \mathcal{D}_{i}\right)$,

(6) $P_{j}^{\mathrm{b}}$ forces that $\wp\left(\mathbb{N}, P_{i}^{\mathrm{a}}\right)$ is not a splitting family.

Proof. Let $\mathrm{a} \in \mathrm{APv}$ and $\kappa \leq\left|P_{\kappa}^{\mathrm{a}}\right|^{\aleph_{0}} \leq \theta<\lambda$. If needed, we can first extend a by applying Lemma1.8, so as to assume that $\Vdash_{P_{i}^{a}} \mathfrak{c}=\theta$ for all $i<\kappa$. Before proceeding we note that it follows from Proposition 1.8 and Lemma 5.11, that condition (4) will imply that $\left\{P_{i}^{\mathrm{b}}: i<\kappa\right\}$ is a continuous $<$--chain of ccc posets as required in the definition of APv. We construct the sequence $\left\{\mathcal{D}_{i}: i<\kappa\right\}$ by recursion on $i<\kappa$. It follows from Lemma 5.20 that there is a set $\mathcal{D}_{0} \subset \wp\left(\mathcal{L}_{1}, P_{0}^{\mathrm{a}} * \mathrm{Fn}(\{0\} \times \theta \times \mathbb{N}, 2)\right)$ such that $\{\emptyset\} \times \mathcal{D}_{0}$ is forced to be a subset of $\mathcal{P}_{\text {Bould }}$ that is $\aleph_{1}$-directed mod finite. Assume that $\overline{1}<\kappa$ and that $\left\{\mathcal{D}_{i}: i<\overline{1}\right\}$ has been constructed so that for $i<j<\overline{1}$, properties (2)-(6) hold and so that $P_{i+1}^{\mathrm{b}}$ forces that $\mathcal{A}_{i}^{\mathrm{a}}$ is very thin over the forcing extension by $P_{i}^{\mathrm{b}}$. It will be most convenient to continue the argument in a forcing extension.

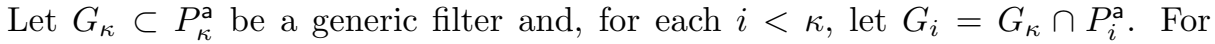
each $i<\kappa$, let $H_{i} \subset \operatorname{Fn}(i+1 \times \theta \times \mathbb{N}, 2)$ be a filter so that $G_{i} \times H_{i}$ is a generic filter for $P_{i}^{\mathrm{a}} * \operatorname{Fn}(i+1 \times \theta \times \mathbb{N}, 2)$. It follows that $\bar{G}=G_{\overline{1}} *\left(\bigcup\left\{H_{i}: i<\overline{1}\right\}\right)$ is a generic filter for $P_{\overline{\mathrm{i}}}^{\mathrm{a}} * \operatorname{Fn}(\overline{\mathrm{i}} \times \theta \times \mathbb{N}, 2)$. We work in the forcing extension $V[\bar{G}]$. We first handle the case when $\overline{1}$ is a limit ordinal. By the definition of the family APv, the sequence $\left\{\mathcal{A}_{i}^{\text {a }}: i<\kappa\right\}$ is not a concern, as in Definition 2.2, when defining $P_{\overline{1}}^{\mathrm{b}}$ in the limit case. It should be clear that the $\bar{G}$-interpretation of the collection $\mathcal{E}=\bigcup\left\{\{\emptyset\} \times \mathcal{D}_{i}: i<\overline{1}\right\}$ is a finitely directed subset of $\mathcal{P}_{\text {Bould }}$. We proceed as in the base case. By Lemma [5.20, there is a $\operatorname{Fn}(\{\overline{1}\} \times \theta \times \mathbb{N}, 2)$-name, $\dot{\mathcal{E}}^{\prime}$, of a subset of $\mathcal{P}_{\text {Bould }}$ that is forced to be an $\aleph_{1}$-directed mod finite extension of $\mathcal{E}$ that further forcing by $Q\left(\dot{\mathcal{E}}^{\prime}\right)$ ensures that the family $[\mathbb{N}]^{\aleph_{0}} \cap V[\bar{G}]$ is not a splitting family. The family $\mathcal{D}_{\overline{1}}$ is a subset of $\wp\left(\mathcal{L}_{1}, P_{\overline{1}}^{\mathrm{a}} * \mathrm{Fn}(\overline{\mathrm{l}}+1 \times \theta \times \mathbb{N}, 2)\right)$ that contains $\mathcal{D}_{i}$ for all $i<\overline{1}$ and is forced to satisfy that $\dot{\mathcal{E}}^{\prime}$ is equal to $\left\{\{\emptyset\} \times T: T \in \mathcal{D}_{\overline{1}}\right\}$.

Now we may assume that $\overline{1}=i+1$ and we note that $\mathcal{A}_{i}^{\text {a }}$ is a family of $P_{i+1}^{\text {a }}$ names that is forced to be very thin over the forcing extension by $P_{i}^{a}$. It follows from Lemma 1.8 that $\mathcal{A}_{i}^{\text {a }}$ is forced to be very thin over the model $V\left[G_{i} * H_{i}\right]$. We again work in the forcing extension $V[\bar{G}]$ where $\bar{G}=G_{i+1} * H_{i}$. Let $\mathcal{A}$ denote the ideal generated by the $\bar{G}$ interpretations of the names from $\mathcal{A}_{i}^{\text {a }}$. Let $\mathcal{E}$ denote the $\aleph_{1-}$ directed mod finite family $\left\{\{\emptyset\} \times T: T \in \mathcal{D}_{i}\right\}$. Let $\dot{x}_{0}$ denote the canonical subset of $\mathbb{N}$ added by $\operatorname{Fn}(\{(i+1,0)\} \times \mathbb{N}, 2)$ over the model $V[\bar{G}]$ as in Definition 1.2. Let $\left\{\dot{n}_{m}\right.$ : $m \in \omega\}$ denote the name of the increasing enumeration of $\dot{x}_{0}$. For all $a \in \mathcal{A}$, let $\dot{I}(a)$ be a canonical $\operatorname{Fn}(\{(i+1,0)\} \times \mathbb{N}, 2)$-name for the set $\left\{m \in \mathbb{N}: a \cap\left[\dot{n}_{m}, \dot{n}_{m+1}\right]=\emptyset\right\}$. For each $q \in Q(\mathcal{E})$, let $\dot{J}(a, q)$ be a canonical $\operatorname{Fn}(\{(i+1,0)\} \times \mathbb{N}, 2)$-name for the set $\left\{\ell \in \mathbb{N}:(\exists m \in \dot{I}(a)) \dot{n}_{m} \leq \max \left(\operatorname{int}\left(t_{\ell-1}\right)\right)<\min \left(\operatorname{int}\left(t_{\ell+1}\right)\right) \leq \dot{n}_{m+1}\right\}$.

Claim 2. The family $\mathcal{E}_{0}=\left\{\left(\emptyset,\left\{t_{\ell}^{q}: \ell \in \dot{J}(a, q)\right\}\right): a \in \mathcal{A}, q \in Q(\mathcal{E})\right\}$ is forced to be a finitely directed subset of $\mathcal{P}_{\text {Bould }}$. 
Proof of Claim: Each $q \in \mathcal{E}$ is in the model $V\left[G_{i} * H_{i}\right]$ and each $a \in \mathcal{A}$ is thin over that model. Therefore there is an infinite set of $\ell \in \mathbb{N}$ such that $a$ is disjoint from $\left[\max \left(\operatorname{int}\left(t_{\ell-1}\right)\right), \min \left(\operatorname{int}\left(t_{\ell+1}\right)\right)\right]$. This implies, by a simple genericity argument, that $\dot{J}(a, q)$ is forced to be an infinite set for each $a \in \mathcal{A}$ and $q \in \mathcal{E}$. Let $H$ be the generic filter for $\operatorname{Fn}(\{(i+1,0)\} \times \mathbb{N}, 2)$ that is equal to $H_{i+1} \cap \operatorname{Fn}(\{(i+1,0)\} \times \mathbb{N}, 2)$. For all $a \in \mathcal{A}$ and $q \in \mathcal{E}$, let $I(a)$ and $J(a, q)$ denote the interpretations by $H$ of $\dot{I}(a)$ and $\dot{J}(a, q)$ respectively. Similarly let $\left\{n_{m}: m \in \mathbb{N}\right\}$ denote the increasing enumeration of the interpretation of $\dot{x}_{0}$.

Evidently, if $a_{1} \subset a_{2}$ are elements of $\mathcal{A}$, then $I\left(a_{2}\right)$ is a subset of $I\left(a_{1}\right)$. To prove the claim it suffices to assume that if $q_{2} \geq q_{1}$ are in $\mathcal{E}$, then, for each $\ell \in J\left(a, q_{2}\right), t_{\ell}^{q_{2}}$ is built from a finite subset of $\left\{t_{k}^{q_{1}}: k \in J\left(a, q_{1}\right)\right\}$. Fix any $\ell \in J\left(a, q_{2}\right)$ and choose minimal finite subsets $B_{\ell-1}, B_{\ell}$ and $B_{\ell+1}$ of $\omega$ such that, for each $r \in\{-1,0,1\}$, $t_{\ell+r}^{q_{2}}$ is built from $\left\{t_{k}^{q_{1}}: k \in B_{\ell+r}\right\}$. Let $k_{0}$ be the maximum element of $B_{\ell-1}$ and let $k_{1}$ be the minimum element of $B_{\ell+1}$. From the definition of $\mathcal{Q}_{\text {Bould }}$, we have, for each $k \in B_{\ell}$, that

(1) $k_{0}<k<k_{1}$,

(2) $\max \left(\operatorname{int}\left(t_{\ell-1}^{q_{2}}\right)\right) \leq \max \left(\operatorname{int}\left(t_{k_{0}}^{q_{1}}\right)\right) \leq \max \left(\operatorname{int}\left(t_{k-1}^{q_{1}}\right)\right)$, and

(3) $\min \left(\operatorname{int}\left(t_{k+1}^{q_{1}}\right)\right) \leq \min \left(\operatorname{int}\left(t_{k_{1}}^{q_{1}}\right)\right) \leq \min \left(\operatorname{int}\left(t_{\ell-1}^{q_{2}}\right)\right)$.

Fix the unique $m \in I(a)$ such that $n_{m} \leq \max \left(\operatorname{int}\left(t_{\ell-1}^{q_{2}}\right)\right)<\max \left(\operatorname{int}\left(t_{\ell+1}^{q_{2}}\right)\right)<n_{m+1}$, and now conclude that $n_{m} \leq \max \left(\operatorname{int}\left(t_{k-1}^{q_{1}}\right)\right)<\max \left(\operatorname{int}\left(t_{k+1}^{q_{1}}\right)\right)<n_{m+1}$. This proves that $B_{\ell} \subset J\left(a, q_{1}\right)$ as required.

Let $\widetilde{H}$ be the generic filter $H_{i+1} \cap \operatorname{Fn}(\{i+1\} \times \theta \times \mathbb{N}, 2)$. For each $q \in Q(\mathcal{E})$ and $a \in \mathcal{A}$, let $q(a) \in Q\left(\mathcal{E}_{0}\right)$ denote the condition $\left(u_{q},\left\{t_{\ell}^{q}: \ell \in J(a, q)\right\}\right)$.

Claim 3. In the forcing extension $V[\bar{G} * \widetilde{H}]$ there is a family $\mathcal{E}_{1} \subset \mathcal{P}_{\text {Bould }}$ such that

(1) $\mathcal{E}_{0} \cup \mathcal{E}$ is a subset of $\mathcal{E}_{1}$,

(2) $\mathcal{E}_{1}$ is $\aleph_{1}$-directed mod finite,

(3) the family $[\mathbb{N}]^{\aleph_{0}} \cap V[\bar{G}]$ is not a splitting family in the further forcing extension by $Q\left(\mathcal{E}_{1}\right)$,

(4) for each $Q(\mathcal{E})$-name $\dot{f} \in V\left[G_{i} * H_{i}\right]$ of an element of $\mathbb{N}^{\mathbb{N}}$ and each $\dot{f}$-ready $q \in Q(\mathcal{E}), q(a) \Vdash_{Q\left(\mathcal{E}_{1}\right)}(\exists n) a \cap[n, \dot{f}(n)]=\emptyset$ for each $a \in \mathcal{A}$.

Proof of Claim: We simply apply Lemma 5.20 to select $\mathcal{E}_{1}$. This ensures that conditions (1)-(3) hold. Now we verify that (4) holds. Let $\dot{f}, a$ and $q$ be as in the statement of $(4)$. Let $r \in Q\left(\mathcal{E}_{1}\right)$ be any condition stronger than $q(a)$. Fix any $k$ so that $\max \left(u_{r}\right)<\min \left(i n t\left(t_{k}^{r}\right)\right)$. Since $r \geq q(a)$, there is a finite subset $B$ of $J(a, q)$ such that $t_{k}^{r}$ is built from $\left\{t_{\ell}^{q}: \ell \in B\right\}$. Choose any $\ell \in B$ such that $e=\operatorname{int}\left(t_{k}^{r}\right) \cap \operatorname{int}\left(t_{\ell}^{q}\right)$ is $t_{\ell}^{q}$-positive. Since $\ell \in J(a, q)$, there is an $m \in I(a)$ so that $n_{m} \leq \max \left(\operatorname{int}\left(t_{\ell-1}^{q}\right)\right)<\min \left(\operatorname{int}\left(t_{\ell+1}^{q}\right)\right)<n_{m+1}$. Since $q$ is $\dot{f}$-ready, there is a $w_{e} \subset e$ such that $\left(u_{r} \cup w_{e},\left\{t_{j}^{q}: \ell<j \in \omega\right\}\right)$ forces that $\dot{f}\left(n_{m}\right)<\min \left(\operatorname{int}\left(t_{\ell+1}^{q}\right)\right)$. Since $m \in I(a)$, this completes the proof that $r$ has an extension forcing that $a$ is disjoint from $\left[n_{m}, \dot{f}\left(n_{m}\right)\right]$.

The proof of the Corollary is completed by choosing a subset $\mathcal{D}_{\overline{1}}$ of $\wp\left(\mathcal{L}_{1}, P_{\overline{1}}^{a} *\right.$ $\operatorname{Fn}(\overline{\mathrm{l}}+1 \times \theta \times \mathbb{N}, 2))($ recall that $\overline{\mathrm{l}}=i+1)$ so that $\mathcal{D}_{i} \subset \mathcal{D}_{\overline{\mathrm{l}}}$ and $\left\{\{\emptyset\} \times T: T \in \mathcal{D}_{\overline{\mathrm{i}}}\right\}$ is forced to equal $\mathcal{E}_{1}$. We prove that $P_{i+1}^{\mathrm{b}}$ forces that $\mathcal{A}_{i}$ is very thin over the forcing extension by $P_{i}^{\mathrm{b}}$. Recall that $G_{i+1}$ is a $P_{i+1}^{\mathrm{a}}$-generic filter and, similarly, $G_{i}=G_{i+1} \cap P_{i}^{\text {a }}$ is $P_{i}^{\text {a }}$-generic. Let $\mathcal{E}_{i}$ denote the interpretation of $\{\emptyset\} \times \mathcal{D}_{i}$, and 
similarly, let $\mathcal{E}_{i+1}$ denote the interpretation of $\{\emptyset\} \times \mathcal{D}_{i+1}$. We already know that $\mathcal{A}_{i}$ is forced to be very thin over the model $V\left[G_{i} * H_{i}\right]$, so it suffices to consider a $Q\left(\mathcal{E}_{i}\right)$-name $\dot{f}$ in $V\left[G_{i} * H_{i}\right]$ of an element of $\mathbb{N}^{\mathbb{N}}$. By Lemma $[5.22$, the set of $\dot{f}$-ready conditions is a dense subset of $Q\left(\mathcal{E}_{i}\right)$. Since $\mathcal{E}_{i}$ is $\aleph_{1}$-directed mod finite, we also have, by Proposition 5.11, that the set of $\dot{f}$-ready conditions from $Q\left(\mathcal{E}_{i}\right)$ is a pre-dense subset of $Q\left(\mathcal{E}_{i+1}\right)$. The result now follows from item (4) of Claim 3.

Definition 5.24. For any $\mathrm{a} \in \mathrm{APv}$ we will say that $\mathrm{b} \in \mathrm{APv}$ is a $Q(\overrightarrow{\mathcal{D}})$-extension of a if there is cardinal $\theta<\lambda$ with $\left|P_{\kappa}^{\mathrm{a}}\right| \leq \theta=\theta^{\aleph_{0}}$ and a sequence $\left\{\mathcal{D}_{i}: i<\kappa\right\}$ such that for all $i<j<\kappa$,

(1) $\mathrm{a}_{1} \leq_{\mathrm{AP}}^{0} \mathrm{~b}$ where $\mathrm{a}_{1}$ is the Cohen ${ }^{\theta}$-extension of $\mathrm{a}$,

(2) $\mathcal{A}_{i}^{\mathrm{a}}=\mathcal{A}_{i}^{\mathrm{b}}$

(3) $\mathcal{D}_{i} \subset \wp\left(\mathcal{L}_{1}, P_{i}^{\mathrm{a}}\right)$,

(4) $\{\emptyset\} \times \mathcal{D}_{i}$ is forced by $P_{i}^{\mathrm{a}_{1}}$ to be an $\aleph_{1}$-directed subset of $\mathcal{P}_{\text {Bould }}$,

(5) $\mathcal{D}_{i} \subset \mathcal{D}_{j}$,

(6) $P_{i}^{\mathrm{b}}=P_{i}^{\mathrm{a}_{1}} * Q\left(\{\emptyset\} \times \mathcal{D}_{i}\right)$,

(7) $P_{j}^{\mathrm{b}}$ forces that $\wp\left(\mathbb{N}, P_{i}^{\mathrm{a}}\right)$ is not a splitting family.

Now we formulate the APv version of Theorem 4.1

$$
\text { 6. } \pi p(\mathfrak{U}) \leq \kappa=\mathfrak{b} \text { AND } \mathfrak{s}=\lambda
$$

Fix, as in Section 4, a 1-to-1 function $h$ from $\lambda$ onto $H(\lambda)$. Recall that by our assumption $\operatorname{Hyp}(\kappa, \lambda), E$ is a stationary subset of $S_{\kappa}^{\lambda}$ and $\left\{C_{\alpha}: \alpha \in \lambda\right\}$ is $\square$-sequence. We let $\left\{X_{\alpha}: \alpha \in E\right\}$ be the $\diamond$-sequence on $\lambda$ as postulated in by $\operatorname{Hyp}(\kappa, \lambda)$.

Theorem 6.1. Assume Hyp $(\kappa, \lambda)$. There is a sequence $\left\{\mathrm{a}_{\alpha}, \zeta_{\alpha}: \alpha \in \lambda\right\}$ such that for each limit $\delta \in \lambda$ :

(1) the sequence $\left\{\mathrm{a}_{\alpha}: \alpha<\delta\right\}$ is $\leq_{\mathrm{AP}}^{*}$-increasing subset of $\mathrm{APv}$,

(2) $\left\{\zeta_{\alpha}: \alpha<\delta\right\} \subset \lambda$ is non-decreasing, and $\zeta_{\delta} \in \lambda$ is the supremum,

(3) if $\delta \notin E$, the sequence $\left\{\mathrm{a}_{\alpha}: \alpha \in \operatorname{acc}\left(C_{\delta}\right) \cup\{\delta\}\right\}$ is a $\leq_{\mathrm{AP}}^{0}$-increasing continuous chain,

(4) if $\delta \in E$ and $\mathcal{E}_{\delta}=\left\{h(\xi): \xi \in X_{\delta}\right\}$ is a maximal subset of $\wp\left(\mathbb{N}, P_{\kappa}^{\mathrm{a} \delta}\right)$ that is forced by $P_{\kappa}^{a_{\delta}}$ to be a free ultrafilter on $\mathbb{N}$, then $\mathcal{E}_{\delta} \cap \mathcal{A}_{i}^{\text {as }}$ is not empty for all $i<\kappa$,

(5) $\mathrm{a}_{\delta+1}$ is the Cohen ${ }^{\omega_{1}}$-extension of $\mathrm{a}_{\delta}$ and $\zeta_{\delta+1}=\zeta_{\delta}$,

(6) if $\alpha=\delta+1$ then $\zeta_{\alpha+1}=\zeta_{\alpha}$ and $\mathrm{a}_{\alpha+1}$ is a Cohen ${ }^{\theta_{\alpha}}$-extension of $\mathrm{a}_{\alpha}$ where $\theta_{\alpha}=\left|P_{\kappa}^{\mathrm{a}_{\alpha}}\right|^{\aleph_{0}}$

(7) if $\alpha=\delta+2$, then $\zeta_{\alpha+1}=\zeta_{\alpha}$ and $\mathrm{a}_{\alpha+1}$ is a $Q(\overrightarrow{\mathcal{D}})$-extension of $\mathrm{a}_{\delta}$,

(8) if $\alpha \in(\delta+2, \delta+\omega)$, then $\zeta_{\alpha+1}$ is the minimal value strictly above $\zeta_{\alpha}$ such that $\dot{Q}_{\alpha+1}=h\left(\zeta_{\alpha+1}-1\right)$ has cardinality less than $\kappa$ and is a $P_{\kappa}^{\mathrm{a}_{\alpha}}$-name of a poset that is forced to be ccc, and $\mathrm{a}_{\alpha+1}=\mathrm{a}_{\alpha} * \dot{Q}_{\alpha+1}$ as in Definition 2.12,

Proof. The proof only involves very minor modifications to the proof of Theorem 4.1 and can be omitted.

Theorem 6.2. Assume $\operatorname{Hyp}(\kappa, \lambda)$. There is a ccc poset $P$ forcing that $\mathfrak{s}=\mathfrak{b}=\lambda$, $M A(\kappa)$, and $\pi p(\mathcal{U}) \leq \kappa$ for all free ultrafilters $\mathcal{U}$ on $\mathbb{N}$. 
Proof. Let $\left\{\mathrm{a}_{\alpha}, \zeta_{\alpha}: \alpha \in \lambda\right\}$ be the sequence constructed in Theorem 4.1. Let $P$ be the poset $\bigcup\left\{P_{\kappa}^{a_{\alpha}}: \alpha \in \lambda\right\}$. Since $\left\{P_{\kappa}^{a_{\alpha}}: \alpha \in \lambda\right\}$ is a strongly continuous <-increasing chain of ccc posets, it follows that $P$ is ccc. Furthermore $\wp(\mathbb{N}, P)$ is equal to the union of the increasing sequence $\left\{\wp\left(\mathbb{N}, P_{\kappa}^{a_{\alpha}}\right): \alpha<\lambda\right\}$. It then follows immediately from condition (7) that $\mathfrak{s}$ is forced to be $\lambda$. By Proposition 2.8, $P$ forces that $\mathfrak{b} \leq \kappa$. The fact that $P$ forces that $\mathfrak{b}=\kappa$ follows once we we note that $P$ forces that $M A(\kappa)$ holds. This is proven exactly as in the proof of Theorem 4.2 and so can be omitted. Similarly, simply repeating that portion of the proof from Theorem 4.2 also proves that $P$ forces that $\pi p(\mathcal{U}) \leq \kappa$ for all free ultrafilter $\mathcal{U}$ on $\mathbb{N}$.

\section{REFERENCES}

[1] Uri Abraham, Proper forcing, Handbook of set theory. Vols. 1, 2, 3, Springer, Dordrecht, 2010, pp. 333-394.

[2] James E. Baumgartner and Peter Dordal, Adjoining dominating functions, J. Symbolic Logic 50 (1985), no. 1, 94-101, DOI 10.2307/2273792. MR780528 (86i:03064)

[3] Andreas Blass and Saharon Shelah, Ultrafilters with small generating sets, Israel J. Math. 65 (1989), no. 3, 259-271, DOI 10.1007/BF02764864. MR1005010 (90e:03057)

[4] Jörg Brendle, The almost-disjointness number may have countable cofinality, Trans. Amer. Math. Soc. 355 (2003), no. 7, 2633-2649 (electronic), DOI 10.1090/S0002-9947-03-03271-9. MR1975392 (2004c:03062)

[5] Jörg Brendle and Vera Fischer, Mad families, splitting families and large continuum, J. Symbolic Logic 76 (2011), no. 1, 198-208, DOI 10.2178/jsl/1294170995. MR2791343 (2012d:03113)

[6] Jörg Brendle and Saharon Shelah, Ultrafilters on $\omega$-their ideals and their cardinal characteristics, Trans. Amer. Math. Soc. 351 (1999), no. 7, 2643-2674, DOI 10.1090/S0002-994799-02257-6. MR1686797 (2000m:03111)

[7] R. Michael Canjar, On the generic existence of special ultrafilters, Proc. Amer. Math. Soc. 110 (1990), no. 1, 233-241. MR993747

[8] Kenneth Kunen and Jerry E. Vaughan (eds.), Handbook of set-theoretic topology, NorthHolland Publishing Co., Amsterdam, 1984. MR776619 (85k:54001)

[9] Vera Fischer and Juris Steprāns, The consistency of $\mathfrak{b}=\kappa$ and $\mathfrak{s}=\kappa^{+}$, Fund. Math. 201 (2008), no. 3, 283-293, DOI 10.4064/fm201-3-5. MR2457482 (2009j:03078)

[10] Saharon Shelah, The character spectrum of $\beta(\mathbb{N})$, Topology Appl. 158 (2011), no. 18, 25352555, DOI 10.1016/j.topol.2011.08.014. MR2847327

[11] _ On cardinal invariants of the continuum, Axiomatic set theory (Boulder, Colo., 1983), Contemp. Math., vol. 31, Amer. Math. Soc., Providence, RI, 1984, pp. 183-207, DOI 10.1090/conm/031/763901. MR763901 (86b:03064)

Department of Mathematics, University of North Carolina at Charlotte, CharLOTTE, NC 28223

E-mail address: adow@uncc.edu

Department of Mathematics, Rutgers University, Hill Center, Piscataway, New JerSEY, U.S.A. 08854-8019

Current address: Institute of Mathematics, Hebrew University, Givat Ram, Jerusalem 91904, Israel

E-mail address: shelah@math.rutgers.edu 